\title{
Conflicting nonlinearities and spectral lacunae bounded on one side by eigenvalues
}

Hans-Jörg Ruppen

Cours de Mathématiques Spéciales, Ecole Polytechnique Fédérale de Lausanne, Station 4, 1015 Lausanne, Switzerland

(hans-joerg.ruppen@epfl.ch)

(MS received 12 March 2004; accepted 23 March 2005)

This paper deals with eigenvalue problems of the form

$$
\begin{aligned}
-u^{\prime \prime}(x)+V(x) u(x) & -q(x)|u(x)|^{\sigma} u(x) \\
& +\mu r(x)|u(x)|^{\tau} u(x)=\lambda u(x), \quad x \in \mathbb{R}, u \in H^{1}(\mathbb{R}) \backslash\{0\} .
\end{aligned}
$$

where $0<\sigma<\tau$ and $V(x)$ is such that the spectrum of $-u^{\prime \prime}$ consists of eigenvalues $\lambda_{1}, \lambda_{2}, \ldots$ situated below the continuous spectrum $[\Lambda,+\infty[$.

We analyse the existence of (multiple) solutions for $\lambda<\lambda_{1}$ as well as for $\lambda>\lambda_{1}$ when $\lambda$ is in a spectral lacuna.

The existence of solutions depends on the weight of $\mu>0$. Moreover, when $\lambda$ increases (while $\mu$ is kept fixed), some solutions are lost when crossing eigenvalues.

The above results are derived with the help of an abstract approach based on variational techniques for multiple solutions. This approach can even be applied to a wider class of problems, the one presented herein being only a model problem.

\section{Introduction}

We consider in this paper nonlinear eigenvalue problems of the following kind.

Problem 1.1. Find $\lambda \in \mathbb{R}$ and $u \in H^{1}(\mathbb{R}) \backslash\{0\}$ such that

$$
-u^{\prime \prime}(x)+V(x) u(x)-q(x)|u(x)|^{\sigma} u(x)+\mu r(x)|u(x)|^{\tau} u(x)=\lambda u(x), \quad x \in \mathbb{R},
$$

holds (in a generalized sense).

It will be assumed that $V(\cdot)$ is a non-negative function, that $q(\cdot)$ and $r(\cdot)$ are positive functions and that $0<\sigma<\tau$. The positive parameter $\mu$ measures the strength of the second nonlinear term; indeed we may assume without loss of generality that $|r|_{L^{\infty}}=1$, for example.

Nonlinear eigenvalue problems of this kind have been studied very intensively by many authors.

A first class of publications covers the case where $V(\cdot) \equiv 0$ and $\mu=0$. Nowadays, it is well known that, for each $\lambda<0$, problem 1.1 has an infinite number of solutions $[4,5,12,16-20,23-28]$, this being so under rather general assumptions on $q(\cdot)$ and $\sigma$.

Concerning the case of Schrödinger equations (where $V(\cdot) \not \equiv 0$ ), we refer the reader to $[3,6,9,13,15,29]$. 


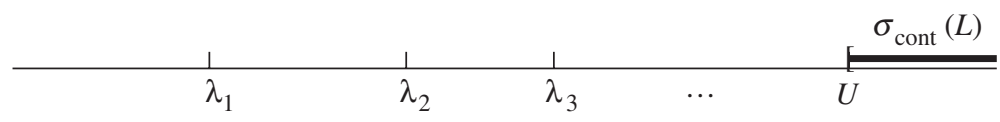

Figure 1. Spectrum of $\sigma(L)$.

We have analysed problem 1.1 with $\mu>0$ and for $\lambda$ below the spectrum of the linearization under such general assumptions in [21]. The key results can be summarized by the observation that the nonlinear terms

$$
q(x)|u(x)|^{\sigma} u(x) \quad \text { and } \quad \mu r(x)|u(x)|^{\tau} u(x)
$$

are conflicting in the sense that the first term gives rise to solutions, while the second term prohibits the existence of solutions. More precisely, we could establish that, for a fixed value of $\lambda$ below the spectrum of the linearization, our problem 1.1 has no solutions at all as soon as $\mu>0$ is sufficiently large, while there are solutions for $\mu>0$ sufficiently small. We insist on the fact that the threshold value of $\mu$ appearing here depends on $\lambda$ : solutions exist for a given $\lambda$ below the spectrum as long as $\mu \in[0, c(\lambda))$, where $c(\cdot)$ is a non-decreasing function and there are no solutions for $\mu>c(\lambda)$.

In [21], we established another important fact. By symmetry it is obvious that solutions appear pairwise in the form $(\lambda, \pm u)$. For $\mu>0$ sufficiently small, we could associate with each solution pair $(\lambda, \pm u)$ a second solution pair $(\lambda, \pm v)$ that has no equivalence in the case $\mu=0$. Thus, we are led to the interesting fact that the conflict of nonlinear terms is in fact solution-generating as long as the conflicting terms are well equilibrated, i.e. $\mu>0$ is not too large. Among authors that have dealt with such situations let us cite [1].

A second class of publications covers the case where $V(\cdot) \not \equiv 0$, but where still $\mu=0$. In this case, the values of $\lambda$ lying in a spectral gap of the linear operator appearing in problem 1.1 have been studied with special interest in the last few years (see, for example, $[7,8,10,11])$. At first glance, it seems that a generalization to the case where $\mu>0$ should be easily realized and that the results cited above should be recovered when $\mu \neq 0$. It turns out that the situation is somewhat more complicated.

The first reason for this is the fact that the above-mentioned equilibrium of the nonlinear terms requires a loss of compactness for the nonlinear potential

$$
\Psi(u)=\frac{1}{2+\tau} \int_{\mathbb{R}} r(x)|u(x)|^{2+\tau} \mathrm{d} x ;
$$

this in turn makes it more difficult to handle the compactness condition of PalaisSmale type.

The second reason is less technical, since solutions $\left(\lambda, \pm u_{\lambda}\right)$ may be lost while crossing with $\lambda$ eigenvalues of the linear part in problem 1.1. In order to keep this loss of solutions sufficiently small, we will have to assume that the spectral gap under consideration will be bounded on the left only by eigenvalues. This means roughly that the spectrum $\sigma(L)$ of the linear part $-u^{\prime \prime}(x)+V(x) u(x)$ can be split into the point spectrum $\sigma_{p}(L)=\left\{\lambda_{i} \mid i=1,2,3, \ldots\right\}$ and the continuous spectrum $\sigma_{\text {cont }}(L)$ according to figure 1 . 
The paper is organized as follows. In $\S 2$ we give an abstract formulation of our problem. This abstract problem covers the situation discussed here, but it covers other problems too. The abstract variational formulation needs some kind of a compactness property, a topic developed in $\S 3$. After a remark on the nonexistence of solutions for small, negative values of $\lambda$ (say $\lambda<\Lambda$ ), we analyse the existence of solutions for $\lambda>\Lambda$. In order to achieve this, we first identify (in $\S 4$ ) two critical sets $\mathfrak{N}_{\lambda}$ and $\mathfrak{M}_{\lambda}$ containing all the non-trivial solutions. A solution $w_{1, \lambda}$ below the spectrum can be found by minimization over $\mathfrak{N}_{\lambda}$; with a mountain-pass argument (over $\mathfrak{M}_{\lambda}$ ) we can find a second solutions $v_{1, \lambda}$ (see $\S 4$ ). In $\S 5$, we derive the existence of more solutions by replacing, for example, minimization over $\mathfrak{N}_{\lambda}$ by a Ljusternik-Schnirelmann argument. Moreover, we discuss how solutions are lost when increasing $\lambda$ across an eigenvalue. In the last section we formulate the result for the concrete problem mentioned above (see figure 7 on p. 1070).

\section{The abstract problem}

We now introduce the abstract problem we will deal with throughout this paper: we are looking for $\lambda \in \mathbb{R}$ and $u \in H \backslash\{0\}$ such that

$$
L u-F(u)+\mu G(u)=\lambda u
$$

holds in some appropriate space and in a sense to be made more precise. The setting of this problem will be rather general, since we make only the following assumption.

Assumption 2.1. $H$ is a separable, real Hilbert space with scalar product $(\cdot, \cdot)$ and associated norm $\|\cdot\|$.

The operators $F$ and $G$ are nonlinear, while $L$ is a linear operator satisfying the following assumption.

Assumption 2.2. $L: \boldsymbol{D}(L) \subset H \rightarrow H$ is a linear, self-adjoint operator that is bounded from below:

$$
\exists \ell>0 \text { such that }(L u, u) \geqslant \ell\|u\|^{2}, \quad \forall u \in \boldsymbol{D}(L) .
$$

The spectrum $\sigma(L)$ of $L$ is such that there exist some constants $U>0$ and $\tilde{U}>U$ such that

$$
\sigma(L) \cap]-\infty, \tilde{U}\left[=\sigma_{1} \cup \sigma_{2},\right.
$$

where

(i) $\sigma_{1}=\left\{\lambda_{i} \mid i=1, \ldots, M\right\}$ is composed of all the $M$ eigenvalues $(M \geqslant 1$, with $M=\infty$ possible),

(ii) $\sigma_{2}=\left[U, \tilde{U}\left[\subset \sigma_{\text {cont }}(L)\right.\right.$.

For convenience, we set $\lambda_{M+1}:=U\left(\lambda_{\infty}:=U\right.$ if $\left.M=\infty\right)$ and we note that $\ell=\lambda_{1}$.

In order to give a broad sense to the concept of solution, we introduce some subspaces of $H$. For $s \geqslant 0, H^{s}$ denotes the domain of $L^{s / 2}$ equipped with the scalar product

$$
(u, v)_{s}:=\left(L^{s / 2} u, L^{s / 2} v\right)
$$


and the associated norm $\|u\|_{s}=\left\|L^{s / 2} u\right\|=\sqrt{\left(L^{s} u, u\right)} ; H^{-s}$ denotes the dual space $\left(H^{s}\right)^{\prime}$. Clearly, $H^{0}=H$.

Proposition 2.3. Under assumptions 2.1 and 2.2, the spaces $H^{s}$ and $H^{-s}$ are separable, real Hilbert spaces, for all $s \geqslant 0$.

The spaces $H^{2}, H^{1}, H$ and $H^{-1}$ will be of special interest. We may consider $H^{2}$, $H^{1}$ and $H$ as subspaces of $H^{-1}$ (see [7] for more details).

We denote by $\langle\cdot, \cdot\rangle$ the duality between $H^{-1}$ and $H^{1}$. In particular, for $u \in H$ and $v \in H^{1}$, we have $\langle u, v\rangle=(u, v)$. So, for $u \in H^{2}$ and $v \in H^{1}$, we find, in view of $L u \in H$, that

$$
|\langle L u, v\rangle|=\left|\left(L^{1 / 2} u, L^{1 / 2} v\right)\right| \leqslant\|u\|_{1}\|v\|_{1} .
$$

This means that

$$
L: \boldsymbol{D}(L)=H^{2} \subset H^{1} \rightarrow H^{-1}
$$

is a continuous operator of norm less or equal to 1 . Hence, we can make the following proposition.

Proposition 2.4. Under assumptions 2.1 and 2.2 there exists exactly one continuous extension $L_{1}$ of $L$ :

$$
L_{1}: H^{1} \rightarrow H^{-1}, \quad \text { with } L_{1 \mid H^{2}}=L .
$$

We can now formulate the problem we are dealing with more precisely, as follows.

Problem 2.5. Find $\lambda \in \mathbb{R}$ and $u \in H^{1} \backslash\{0\}$ such that

$$
L_{1} u-F(u)+\mu G(u)=\lambda u
$$

holds in $H^{-1}$.

REMARKS.

(i) $\lambda \in \mathbb{R}$ is an eigenvalue and we are looking for solutions $\left(\lambda, u_{\lambda}\right) \in \mathbb{R} \times H^{1}$. The method we will use consists in finding for a given value of $\lambda$ the corresponding functions $u_{\lambda}$.

(ii) $\mu$ is a positive constant that remains fixed. It is in fact a measure for the amplitude of the term $\mu G$.

(iii) The operators $F, G: H^{1} \rightarrow H^{-1}$ are nonlinear (see below). Let us simply remark for the moment that $F(0)=G(0)=0$. Hence, the aim of the condition $H^{1} \backslash\{0\}$ is to exclude the 'trivial' solutions $(\lambda, 0)$ in problem 2.5.

We now introduce the assumptions made on $F$ and $G$.

Assumption 2.6. There exists $\Phi \in C^{1}\left(H^{1} ; \mathbb{R}\right)$ such that $F=\Phi^{\prime}$. Moreover, we have the following conditions:

(i) $\Phi(0)=0$ and $\Phi(u)>0$ for $u \in H^{1} \backslash\{0\}$;

(ii) $\exists \sigma>0$ such that $\langle F(u), u\rangle=(2+\sigma) \Phi(u), \forall u \in H^{1}$; 
(iii) $\exists c_{F}>0$ and $p_{F}>1$ such that

$$
\|F(u)\|_{-1} \leqslant c_{F}\langle F(u), u\rangle^{1 / p_{F}}, \quad \forall u \in H^{1} .
$$

Assumption 2.7. There exists $\Psi \in C^{1}\left(H^{1} ; \mathbb{R}\right)$ such that $G=\Psi^{\prime}$. Moreover, we have the following conditions:

(i) $\Psi(0)=0$ and $\Psi(u)>0$ for $u \in H^{1} \backslash\{0\}$;

(ii) $\exists \tau>0$ such that $\langle G(u), u\rangle=(2+\tau) \Psi(u), \forall u \in H^{1}$;

(iii) $\exists c_{G}>0$ and $p_{G}>1$ such that

$$
\|G(u)\|_{-1} \leqslant c_{G}\langle G(u), u\rangle^{1 / p_{G}}, \quad \forall u \in H^{1} .
$$

We will see below that these assumptions imply $F(0)=G(0)=0$ and

$$
p_{F}=\frac{2+\sigma}{1+\sigma}, \quad p_{G}=\frac{2+\tau}{1+\tau} .
$$

Until now, we have presented the similar faces of $F$ and $G$. We now introduce an additional assumption on the coexistence of these terms.

Assumption 2.8. The operator $F: H^{1} \rightarrow H^{-1}$ is compact. Moreover, whenever $u_{n} \rightarrow u \in H^{1}$, we have

$$
\begin{gathered}
F\left(u_{n}\right)-\mu G\left(u_{n}\right) \rightarrow F(u)-\mu G(u), \quad F\left(u_{n}\right) \rightarrow F(u) \quad \text { in } H^{-1}, \\
\Phi\left(u_{n}\right) \rightarrow \Phi(u) \quad \text { in } \mathbb{R}, \quad \Psi(u) \leqslant \liminf _{n \rightarrow \infty} \Psi\left(u_{n}\right) .
\end{gathered}
$$

We have, for any sequence $\left\{u_{n}\right\} \subset H^{1}$ with $\lim _{n \rightarrow \infty} \Psi\left(u_{n}\right)=+\infty$,

$$
\lim _{n \rightarrow \infty} \frac{\Phi\left(u_{n}\right)}{\mu \Psi\left(u_{n}\right)}=0
$$

Finally, $0<\sigma<\tau$.

For a discussion of existing relations between these properties we refer the reader to the classical theorems of Tsitlanadze [30]

In order to simplify the notation, we introduce the following shortcut.

Assumption 2.9. The assumptions 2.1, 2.2, 2.6, 2.7 and 2.8 hold.

Within this setting, problem 2.5 is of variational type. Therefore, we introduce the notation:

$$
\begin{aligned}
B(u) & :=\left\langle L_{1} u, u\right\rangle=\left\|L^{1 / 2} u\right\|^{2}=\|u\|_{1}^{2}, \\
B_{\lambda}(u) & :=B(u)-\lambda\|u\|^{2}, \\
I_{\lambda}(u) & :=\frac{1}{2} B_{\lambda}(u)-\Phi(u), \\
J_{\lambda}(u) & :=\frac{1}{2} B_{\lambda}(u)-\Phi(u)+\mu \Psi(u)=I_{\lambda}(u)+\mu \Psi(u) .
\end{aligned}
$$

Then the following proposition holds. 
Proposition 2.10. Under assumption 2.9, we see that

$$
(\lambda, u) \text { solves problem } 2.5 \Longleftrightarrow\left\{\begin{array}{l}
J_{\lambda}^{\prime}(u)=0, \\
u \in H^{1} \backslash\{0\} .
\end{array}\right.
$$

The rest of this section is devoted to the study of terms $B_{\lambda}, \Phi$ and $\Psi$.

Proposition 2.11. Under assumptions 2.1 and 2.6 we have

(1) $\Phi(t u)=t^{2+\sigma} \Phi(u)$ for $t>0, \forall u \in H^{1}$,

(2) $p_{F}=\frac{2+\sigma}{1+\sigma}$ and, for all $u \in H$,

$$
\|F(u)\|_{-1} \leqslant \text { const. }\|u\|_{1}^{1+\sigma} \quad \text { and } \quad \Phi(u) \leqslant \text { const. }\|u\|_{1}^{2+\sigma} .
$$

Proof. We follow Heinz [10]. Concerning the first point, it is sufficient to prove the claim for $u \neq 0$. In this case we have

$$
\begin{aligned}
\log \Phi(t u)-\log \Phi(u) & =\int_{1}^{t} \frac{\langle F(s u), s u\rangle}{s \Phi(s u)} \mathrm{d} s \\
& =(2+\sigma) \int_{1}^{t} \frac{1}{s} \mathrm{~d} s \\
& =(2+\sigma) \log t
\end{aligned}
$$

and the claim follows. For the second point, we note that

$$
\|F(u)\|_{-1} \leqslant c_{F}\langle F(u), u\rangle^{1 / p_{F}} \leqslant c_{F}\|F(u)\|_{-1}^{1 / p_{F}}\|u\|_{1}^{1 / p_{F}}
$$

so that

$$
\|F(u)\|_{-1} \leqslant \text { const. }\|u\|_{1}^{1 /\left(p_{F}-1\right)}=\text { const. }\|u\|_{1}^{p^{\prime}-1},
$$

where $p^{\prime}=p_{F} /\left(p_{F}-1\right)$. Hence,

$$
\begin{aligned}
t^{2+\sigma} \Phi(u) & =\Phi(t u)=\int_{0}^{1}\langle F(s t u), t u\rangle \mathrm{d} s \\
& \leqslant \int_{0}^{1}\|F(s t u)\|_{-1}\|t u\|_{1} \mathrm{~d} s \leqslant \text { const. } t^{p^{\prime}}, \quad \forall t>0,
\end{aligned}
$$

and this implies that $p^{\prime}=2+\sigma$ and $p_{F}=(2+\sigma) /(1+\sigma)$. Moreover,

$$
\Phi(u)=\frac{1}{2+\sigma}\langle F(u), u\rangle \leqslant \frac{1}{2+\sigma}\|F(u)\|_{-1}\|u\|_{1} \leqslant \text { const. }\|u\|_{1}^{2+\sigma} .
$$

So we are done.

By the same arguments we obtain the following proposition.

Proposition 2.12. Under the assumptions 2.1 and 2.7 we have

(i) $\Psi(t u)=t^{2+\tau} \Psi(u)$ for $t>0, \forall u \in H^{1}$; 
(ii) $p_{G}=\frac{2+\tau}{1+\tau}$ and, $\forall u \in H$

$$
\|G(u)\|_{-1} \leqslant \text { const. }\|u\|_{1}^{1+\tau} \quad \text { and } \quad \Psi(u) \leqslant \text { const. }\|u\|_{1}^{2+\tau} .
$$

When analysing the quadratic term $B_{\lambda}(u)$, we restrict our analysis to

$$
\lambda \in]-\infty, \lambda_{M+1}\left[\backslash \sigma\left(L_{1}\right) .\right.
$$

DeFinition 2.13.

(1) If $\lambda<\lambda_{1}$, we set

$$
\begin{aligned}
& \left.\lambda^{-}:=-\infty \text { and } \lambda^{+}:=\lambda_{1}, \text { so that } \lambda \in\right] \lambda^{-}, \lambda^{+}[, \\
& P_{\lambda}^{-}:=0: H \rightarrow H \quad \text { and } \quad P_{\lambda}^{+}:=\text {id }: H \rightarrow H .
\end{aligned}
$$

(2) If $\lambda \in] \lambda_{1}, \lambda_{M+1}\left[\backslash \sigma\left(L_{1}\right)\right.$, we set

$$
\begin{aligned}
& \lambda^{-}:=\max \left\{\lambda_{i} \mid \lambda_{i}<\lambda \text { where } i=1, \ldots, M\right\}, \\
& \lambda^{+}:=\min \left\{\lambda_{i} \mid \lambda_{i}>\lambda \text { where } i=1, \ldots, M+1\right\},
\end{aligned}
$$

so that $\lambda \in] \lambda^{-}, \lambda^{+}\left[\right.$. Moreover, we denote by $P_{\lambda}^{-}: H \rightarrow H$ the orthogonal projection associated with the interval $\left.]-\infty, \lambda^{-}\right]$in the decomposition of the unity of the operator $L$; in a similar way we denote by $P_{\lambda}^{+}: H \rightarrow H$ the orthogonal projection associated with the interval $\left[\lambda^{+},+\infty\left[\right.\right.$. Clearly, $P_{\lambda}^{-}+$ $P_{\lambda}^{+}=\mathrm{id}$.

(3) In any case we define $E_{\lambda}^{ \pm}:=P_{\lambda}^{ \pm}\left(H^{1}\right)$.

LEMMA 2.14. Under assumptions 2.1 and 2.2 and if $\lambda \in]-\infty, \lambda_{M+1}\left[\backslash \sigma\left(L_{1}\right)\right.$, we find that

(i) $P_{\lambda}^{-}$and $P_{\lambda}^{+}$commute with the powers of $L$ in the following sense:

$$
P_{\lambda}^{ \pm} L^{1 / 2} \subset L^{1 / 2} P_{\lambda}^{ \pm}, \ldots
$$

(ii) $E_{\lambda}^{-}$and $E_{\lambda}^{+}$are subspaces of $H^{1}$ such that

$$
H^{1}=E_{\lambda}^{-} \oplus E_{\lambda}^{+},
$$

the sum being orthogonal in $(\cdot, \cdot)$ and $(\cdot, \cdot)_{1}$.

Proposition 2.15. Under assumptions 2.1 and 2.2 and if $\lambda<\lambda_{1}, \sqrt{B_{\lambda}(\cdot)}$ is a norm in $H^{1}$ which is equivalent to the 'usual' norm $B(\cdot)$.

Proof. Indeed,

$$
B(u)=\left[B(u)-\lambda_{1}\|u\|^{2}\right]+\lambda_{1}\|u\|^{2}=B_{\lambda_{1}}(u)+\lambda_{1}\|u\|^{2}
$$

is the usual norm in $H^{1}$ and the claim follows from

$$
\begin{gathered}
B_{\lambda}(u)=B_{\lambda_{1}}(u)+\left(\lambda_{1}-\lambda\right)\|u\|^{2}=B_{\lambda_{1}}(u)+\frac{\lambda_{1}-\lambda}{\lambda_{1}} \lambda_{1}\|u\|^{2}, \\
\min \left\{1, \frac{\lambda_{1}-\lambda}{\lambda_{1}}\right\}\|u\|_{1}^{2} \leqslant B_{\lambda}(u) \leqslant \max \left\{1, \frac{\lambda_{1}-\lambda}{\lambda_{1}}\right\}\|u\|_{1}^{2} .
\end{gathered}
$$


For $\lambda>\lambda_{1}$ with $\lambda \notin \sigma\left(L_{1}\right)$, we set $\beta_{\lambda}(\alpha)=1-\lambda / \alpha$.

Proposition 2.16. Suppose that assumptions 2.1 and 2.2 are fulfilled and that $\lambda \in] \lambda_{1}, \lambda_{M+1}\left[\backslash \sigma\left(L_{1}\right)\right.$. Then

(1) for $\alpha>0$ we have

$$
\begin{aligned}
& B(u) \leqslant \alpha\|u\|^{2} \Longrightarrow B_{\lambda}(u) \leqslant \beta_{\lambda}(\alpha)\|u\|_{1}^{2}, \\
& B(u) \geqslant \alpha\|u\|^{2} \Longrightarrow B_{\lambda}(u) \geqslant \beta_{\lambda}(\alpha)\|u\|_{1}^{2} ;
\end{aligned}
$$

(2) in particular, if $\lambda^{-}<\lambda<\lambda^{+}$, then

$$
\begin{array}{ll}
B_{\lambda}(u) \leqslant \beta_{\lambda}^{-}\|u\|_{1}^{2} & \forall u \in E_{\lambda}^{-}, \text {where } \beta_{\lambda}^{-}:=\beta_{\lambda}\left(\lambda^{-}\right), \\
B_{\lambda}(u) \geqslant \beta_{\lambda}^{+}\|u\|_{1}^{2} & \forall u \in E_{\lambda}^{+}, \text {where } \beta_{\lambda}^{+}:=\beta_{\lambda}\left(\lambda^{+}\right) .
\end{array}
$$

Proof. Concerning the first point, $B(u)=\|u\|_{1} \leqslant \alpha\|u\|^{2}$ implies that $\|u\|^{2} \geqslant$ $(1 / \alpha)\|u\|_{1}^{2}$. So

$$
B_{\lambda}(u)=B(u)-\lambda\|u\|^{2}=\|u\|_{1}^{2}-\lambda\|u\|^{2} \leqslant\|u\|_{1}^{2}-\frac{\lambda}{\alpha}\|u\|_{1}^{2}=\beta_{\lambda}(\alpha)\|u\|_{1}^{2} .
$$

The conclusion for $B(u) \geqslant \alpha\|u\|^{2}$ follows in a similar way. The second point is a consequence of the first point and the remark that

$$
\begin{aligned}
& B(u) \leqslant \lambda^{-}\|u\|^{2}, \quad \forall u \in E^{-}, \\
& B(u) \geqslant \lambda^{+}\|u\|^{2}, \quad \forall u \in E^{+} .
\end{aligned}
$$

\section{The Palais-Smale condition}

When applying variational methods, it is well known that the corresponding potential function $\left(J_{\lambda}\right.$ in the present paper) must verify some compactness condition, such as the Palais-Smale condition. So let us consider some Palais-Smale sequence; by this we mean a sequence $\left\{u_{n}\right\} \subset H^{1}$ such that

$$
\left\{J_{\lambda}\left(u_{n}\right)\right\} \text { is bounded in } H^{1} \text { and }\left\|J_{\lambda}^{\prime}\left(u_{n}\right)\right\|_{-1} \rightarrow 0 \quad \text { as } n \rightarrow+\infty .
$$

We say that the Palais-Smale condition is fulfilled if any such sequence has a convergent subsequence. We analyse in this section the existence of such a converging subsequence.

Combining the equations

$$
\begin{aligned}
J_{\lambda}^{\prime}\left(u_{n}\right) u_{n} & =B_{\lambda}\left(u_{n}\right)-(2+\sigma) \Phi\left(u_{n}\right)+\mu(2+\tau) \Psi\left(u_{n}\right), \\
2 J_{\lambda}\left(u_{n}\right) & =B_{\lambda}\left(u_{n}\right)-2 \Phi\left(u_{n}\right)+\mu 2 \Psi\left(u_{n}\right)
\end{aligned}
$$

leads to the following three basic relations:

$$
\begin{aligned}
2 J_{\lambda}\left(u_{n}\right)-J_{\lambda}^{\prime}\left(u_{n}\right) u_{n} & =\sigma \Phi\left(u_{n}\right)-\mu \tau \Psi\left(u_{n}\right), \\
2(2+\sigma) J_{\lambda}\left(u_{n}\right)-2 J_{\lambda}^{\prime}\left(u_{n}\right) u_{n} & =\sigma B_{\lambda}\left(u_{n}\right)-2(\tau-\sigma) \mu \Psi\left(u_{n}\right), \\
2(2+\tau) J_{\lambda}\left(u_{n}\right)-2 J_{\lambda}^{\prime}\left(u_{n}\right) u_{n} & =\tau B_{\lambda}\left(u_{n}\right)-2(\tau-\sigma) \Phi\left(u_{n}\right) .
\end{aligned}
$$


In particular, we have

$$
\begin{aligned}
\Phi\left(u_{n}\right) & =\frac{\tau}{2(\tau-\sigma)} B_{\lambda}\left(u_{n}\right)-\frac{2+\tau}{\tau-\sigma} J_{\lambda}\left(u_{n}\right)+\frac{1}{\tau-\sigma} J_{\lambda}^{\prime}\left(u_{n}\right) u_{n} \\
\mu \Psi\left(u_{n}\right) & =\frac{\sigma}{2(\tau-\sigma)} B_{\lambda}\left(u_{n}\right)-\frac{2+\sigma}{\tau-\sigma} J_{\lambda}\left(u_{n}\right)+\frac{1}{\tau-\sigma} J_{\lambda}^{\prime}\left(u_{n}\right) u_{n}
\end{aligned}
$$

We distinguish now two cases with respect to the behaviour of $\left|B_{\lambda}\left(u_{n}\right)\right|$.

CASE 1. Suppose that (up to a subsequence) we have $\lim _{n \rightarrow+\infty}\left|B_{\lambda}\left(u_{n}\right)\right| \rightarrow+\infty$.

If the sequence

$$
\left\{\frac{u_{n}}{B_{\lambda}\left(u_{n}\right)}\right\} \subset H^{1}
$$

is bounded, we would have, for $n \rightarrow+\infty$,

$$
J_{\lambda}^{\prime}\left(u_{n}\right) \frac{u_{n}}{B_{\lambda}\left(u_{n}\right)} \rightarrow 0 \quad \text { and } \quad \frac{J_{\lambda}\left(u_{n}\right)}{B_{\lambda}\left(u_{n}\right)} \rightarrow 0,
$$

so that

$$
\Phi\left(u_{n}\right)=B_{\lambda}\left(u_{n}\right)\left[\frac{\tau}{2(\tau-\sigma)}+o(1)\right]
$$

and

$$
\mu \Psi\left(u_{n}\right)=B_{\lambda}\left(u_{n}\right)\left[\frac{\sigma}{2(\tau-\sigma)}+o(1)\right] .
$$

This would imply, for $n \rightarrow+\infty$, that

$$
B_{\lambda}\left(u_{n}\right) \rightarrow+\infty \quad \text { and } \quad \Psi\left(u_{n}\right) \rightarrow+\infty
$$

and thus lead us to the contradiction

$$
\lim _{n \rightarrow+\infty} \frac{\Phi\left(u_{n}\right)}{\mu \Psi\left(u_{n}\right)}=\frac{\tau}{\sigma}>0 .
$$

We may conclude that (up to a subsequence) we have

$$
\frac{\left\|u_{n}\right\|_{1}}{\left|B_{\lambda}\left(u_{n}\right)\right|} \rightarrow+\infty \quad \text { as } n \rightarrow+\infty .
$$

If $\lambda<\lambda_{1}$, this is impossible in view of proposition 2.15 and since

$$
\frac{\left\|u_{n}\right\|_{1}}{\sqrt{B_{\lambda}\left(u_{n}\right)}} \rightarrow+\infty
$$

so that the sequence $\left\{B_{\lambda}\left(u_{n}\right)\right\}$ is in fact bounded.

For $\lambda \in] \lambda_{1}, \lambda_{M+1}[$, the situation calls for a more detailed analysis. To this purpose, we set (taking, if necessary, a subsequence)

$$
\gamma:=\lim _{n \rightarrow+\infty} \frac{J_{\lambda}^{\prime}\left(u_{n}\right) u_{n}}{B_{\lambda}\left(u_{n}\right)}
$$


where $\gamma \in \overline{\mathbb{R}}$. Now $\gamma \in \pm \infty$ would lead us to

$$
\mu \Psi\left(u_{n}\right)=B_{\lambda}\left(u_{n}\right)\left[\frac{\sigma}{2(\tau-\sigma)}+o(1)+\frac{1}{\tau-\sigma} \frac{J_{\lambda}^{\prime}\left(u_{n}\right) u_{n}}{B_{\lambda}\left(u_{n}\right)}\right] \rightarrow+\infty \quad \text { as } n \rightarrow+\infty
$$

and to

$$
\lim _{n \rightarrow+\infty} \frac{\Phi\left(u_{n}\right)}{\mu \Psi\left(u_{n}\right)}=\lim _{n \rightarrow+\infty} \frac{\tau+o(1)+2 J_{\lambda}^{\prime}\left(u_{n}\right) u_{n}\left\{B_{\lambda}\left(u_{n}\right)\right\}^{-1}}{\sigma+o(1)+2 J_{\lambda}^{\prime}\left(u_{n}\right) u_{n}\left\{B_{\lambda}\left(u_{n}\right)\right\}^{-1}}=1>0,
$$

which contradicts assumption 2.8. Hence, $\gamma \in \mathbb{R}$ and

$$
\begin{array}{r}
\Phi\left(u_{n}\right)=\frac{B_{\lambda}\left(u_{n}\right)}{\tau-\sigma}\left[\frac{\tau}{2}+\gamma+o(1)\right], \\
\mu \Psi\left(u_{n}\right)=\frac{B_{\lambda}\left(u_{n}\right)}{\tau-\sigma}\left[\frac{\sigma}{2}+\gamma+o(1)\right] .
\end{array}
$$

We even find that $\gamma \in\left\{-\frac{1}{2} \sigma,-\frac{1}{2} \tau\right\}$. Indeed, $\gamma \neq-\frac{1}{2} \sigma$ implies that $\Psi\left(u_{n}\right) \rightarrow+\infty$ as $n \rightarrow+\infty$, so that

$$
\lim _{n \rightarrow+\infty} \frac{\Phi\left(u_{n}\right)}{\mu \Psi\left(u_{n}\right)}=\frac{\tau+2 \gamma}{\sigma+2 \gamma}=0
$$

shows that $\gamma=-\frac{1}{2} \tau$.

CASE 2. Suppose that the sequence $\left\{\left|B_{\lambda}\left(u_{n}\right)\right|\right\}$ remains bounded.

Note that, in this case, $J_{\lambda}^{\prime}\left(u_{n}\right) u_{n}$ remains bounded, for otherwise (up to some subsequence) we would be led by the relations (3.1) and (3.2) to

$$
J_{\lambda}^{\prime}\left(u_{n}\right) u_{n} \rightarrow+\infty, \quad \Psi\left(u_{n}\right) \rightarrow+\infty \quad \text { and } \quad \lim _{n \rightarrow+\infty} \frac{\Phi\left(u_{n}\right)}{\mu \Psi\left(u_{n}\right)}=1,
$$

a contradiction to assumption 2.8. So, in fact, the sequences

$$
\left\{J_{\lambda}^{\prime}\left(u_{n}\right) u_{n}\right\}, \quad\left\{\Phi\left(u_{n}\right)\right\} \quad \text { and } \quad\left\{\Psi\left(u_{n}\right)\right\}
$$

are all bounded.

Thus, we are led to the following lemma.

Lemma 3.1. Suppose that the assumption 2.9 holds and that $\lambda \in] \lambda_{1}, \lambda_{M+1}[$. Let $\left\{u_{n}\right\}$ be a Palais-Smale sequence. Then, up to a subsequence, we find that either $($ for $n \rightarrow+\infty)$

$$
\begin{aligned}
\left|B_{\lambda}\left(u_{n}\right)\right| & \rightarrow+\infty, \quad \frac{\left\|u_{n}\right\|_{1}}{\left|B_{\lambda}\left(u_{n}\right)\right|} \rightarrow+\infty, \\
\Phi\left(u_{n}\right) & =\frac{B_{\lambda}\left(u_{n}\right)}{\tau-\sigma}\left[\frac{\tau}{2}+\gamma+o(1)\right]
\end{aligned}
$$

and

$$
\mu \Psi\left(u_{n}\right)=\frac{B_{\lambda}\left(u_{n}\right)}{\tau-\sigma}\left[\frac{\sigma}{2}+\gamma+o(1)\right],
$$

where $\gamma \in\{-\tau / 2,-\sigma / 2\}$, or the sequences $\left\{B_{\lambda}\left(u_{n}\right)\right\},\left\{J_{\lambda}^{\prime}\left(u_{n}\right) u_{n}\right\},\left\{\Phi\left(u_{n}\right)\right\}$ and $\left\{\Psi\left(u_{n}\right)\right\}$ are bounded. 
In order to show that $\left\{u_{n}\right\}$ remains bounded in $H^{1}$, we will use the following property, given by assumptions 2.6 and 2.7: $\forall u \in H^{1}$,

$$
\left\|\Phi^{\prime}(u)-\mu \Psi^{\prime}(u)\right\|_{-1} \leqslant \text { const. }\left[\Phi(u)^{(1+\sigma) /(2+\sigma)}+\mu \Psi(u)^{(1+\tau) /(2+\tau)}\right] .
$$

We now obtain, for our Palais-Smale sequence $\left\{u_{n}\right\}$ and for $\lambda \notin \sigma\left(L_{1}\right)$,

$$
\begin{aligned}
\left\|u_{n}\right\|_{1} & =\left\|\left(L_{1}-\lambda\right)^{-1}\left(L_{1}-\lambda\right) u_{n}\right\|_{1} \\
& \leqslant\left\|\left(L_{1}-\lambda\right)^{-1}\right\|\left\|\left(L_{1}-\lambda\right) u_{n}\right\|_{-1} \\
& \leqslant \text { const. }\left[\left\|J_{\lambda}^{\prime}\left(u_{n}\right)\right\|_{-1}+\left\|\Phi^{\prime}\left(u_{n}\right)-\mu \Psi^{\prime}\left(u_{n}\right)\right\|_{-1}\right] \\
& =\text { const. }\left[o(1)+\left\|\Phi^{\prime}\left(u_{n}\right)-\mu \Psi^{\prime}\left(u_{n}\right)\right\|_{-1}\right] .
\end{aligned}
$$

Now suppose for a moment that we find, in the conclusions of lemma 3.1, that $\left|B_{\lambda}\left(u_{n}\right)\right| \rightarrow+\infty$. Then we have

$$
\begin{aligned}
\left\|u_{n}\right\|_{1} & \leqslant \text { const. }\left[o(1)+\text { const. }\left|B_{\lambda}\left(u_{n}\right)\right|^{(1+\tau) /(2+\tau)}\right], \\
\frac{\left\|u_{n}\right\|_{1}}{\left|B_{\lambda}\left(u_{n}\right)\right|} & \leqslant o(1)+\text { const. }\left|B_{\lambda}\left(u_{n}\right)\right|^{-1 /(2+\tau)} \rightarrow 0 .
\end{aligned}
$$

But this is a contradiction.

Hence, $\left|B_{\lambda}\left(u_{n}\right)\right|$ must remain bounded. Then, using the result in lemma 3.1 and (3.3), we are led to

$$
\left\|u_{n}\right\|_{1} \leqslant \text { const., }
$$

i.e. the sequence $\left\{\left\|u_{n}\right\|_{1}\right\}$ is bounded.

Hence, the following proposition holds.

Proposition 3.2. Suppose that assumption 2.9 holds. Suppose moreover that $\lambda \in$ ]$-\infty, \lambda_{M+1}\left[\backslash \sigma\left(L_{1}\right)\right.$. Every Palais-Smale sequence is then bounded in $H^{1}$.

$H^{1}$ is a Hilbert space. So, up to some subsequence, we may assume that, for $n \rightarrow+\infty$,

$$
\begin{aligned}
& u_{n} \rightarrow u \quad \text { in } H^{1}, \quad \Phi^{\prime}\left(u_{n}\right) \rightarrow \Phi^{\prime}(u) \quad \text { and } \quad \Psi^{\prime}\left(u_{n}\right) \rightarrow \Psi^{\prime}(u), \\
& \left(L_{1}-\lambda\right) u_{n} \rightarrow\left(L_{1}-\lambda\right) u \text {. }
\end{aligned}
$$

Now we have

$$
\begin{aligned}
\lim _{n \rightarrow+\infty} 2 J_{\lambda}\left(u_{n}\right) & =\lim _{n \rightarrow+\infty}\left[2 J_{\lambda}\left(u_{n}\right)-J_{\lambda}^{\prime}\left(u_{n}\right) u_{n}\right] \\
& =\lim _{n \rightarrow+\infty}\left[\sigma \Phi\left(u_{n}\right)-\mu \tau \Psi\left(u_{n}\right)\right] \\
& \leqslant \sigma \Phi(u)-\tau \mu \Psi(u) .
\end{aligned}
$$

On the other hand, we have $J_{\lambda}^{\prime}\left(u_{n}\right) \rightarrow 0$ and, since

$$
\begin{aligned}
J_{\lambda}^{\prime}\left(u_{n}\right) & =\left(L_{1}-\lambda\right) u_{n}-\Phi^{\prime}\left(u_{n}\right)+\mu \Psi^{\prime}\left(u_{n}\right) \\
& \rightarrow\left(L_{1}-\lambda\right) u-\Phi^{\prime}(u)+\mu \Psi^{\prime}(u)=J_{\lambda}^{\prime}(u),
\end{aligned}
$$

we obtain $J_{\lambda}^{\prime}(u)=0$. Hence,

$$
\begin{aligned}
\lim _{n \rightarrow+\infty} 2 J_{\lambda}\left(u_{n}\right) & \leqslant \sigma \Phi(u)-\tau \mu \Psi(u) \\
& =2 J_{\lambda}(u)-J_{\lambda}^{\prime}(u) u=2 J_{\lambda}(u) .
\end{aligned}
$$




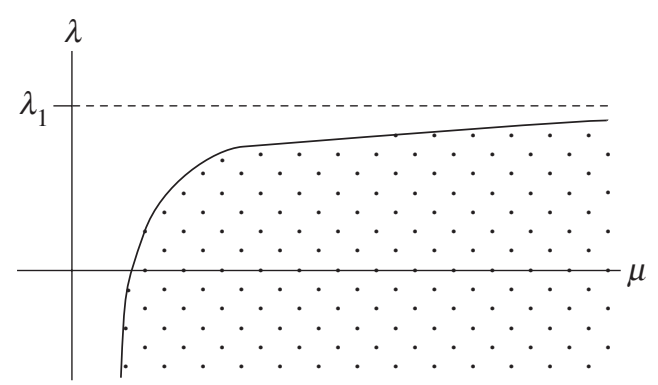

Figure 2. Domain without solutions.

Since

$$
2 J_{\lambda}(u)=\left\langle\left(L_{1}-\lambda\right) u, u\right\rangle-2 \Phi(u)+2 \mu \Psi(u),
$$

we find, up to a subsequence, that

$$
\lim _{n \rightarrow+\infty} B_{\lambda}\left(u_{n}\right) \leqslant B_{\lambda}(u)
$$

If $\lambda<\lambda_{1}$, then, by proposition 2.15, $u_{n} \rightarrow u$ in $H^{1}$. If $\left.\lambda \in\right] \lambda_{1}, \lambda_{M+1}\left[\backslash \sigma\left(L_{1}\right)\right.$, we proceed by decomposition and in this manner we obtain (see lemma 2.14)

$$
B_{\lambda}\left(u_{n}\right)=B_{\lambda}\left(P_{\lambda}^{+} u_{n}\right)+B_{\lambda}\left(P_{\lambda}^{-} u_{n}\right) .
$$

On $E_{\lambda}^{+}$we obtain, by convexity,

$$
\lim _{n \rightarrow+\infty} B_{\lambda}\left(P_{\lambda}^{+} u_{n}\right) \geqslant B_{\lambda}\left(P_{\lambda}^{+} u\right) .
$$

On the other hand, the finite dimensionality of $E_{\lambda}^{-}$implies that

$$
P_{\lambda}^{-}\left(u_{n}\right) \rightarrow P_{\lambda}^{-}(u) \text { in } H^{1}, \quad \lim _{n \rightarrow+\infty} B_{\lambda}\left(P_{\lambda}^{-} u_{n}\right)=B_{\lambda}^{-}(u) .
$$

Thus,

$$
\lim _{n \rightarrow+\infty} B_{\lambda}\left(P_{\lambda}^{+} u_{n}\right)=B_{\lambda}\left(P_{\lambda}^{+} u\right)
$$

and hence $P_{\lambda}^{+}\left(u_{n}\right) \rightarrow P_{\lambda}^{+}(u)$ in $H^{1}$.

Consequently, we obtain the following important result.

Theorem 3.3. Suppose that assumption 2.9 holds and that

$$
\lambda \in]-\infty, \lambda_{M+1}\left[\backslash \sigma\left(L_{1}\right) .\right.
$$

Every Palais-Smale sequence $\left\{u_{n}\right\}$ then has a convergent subsequence.

The above results about the convergence of the Palais-Smale sequence open the door to the variational approaches for the problem under consideration. We close this section with a remark about the nonexistence of solutions. This may be amazing at first glance, but eventually may be seen that the existence of solutions will hold only if $\lambda$ and $\mu$ satisfy some condition that can be thought as an equilibrium between the nonlinearities in our problem.

First, we introduce a new assumption. 


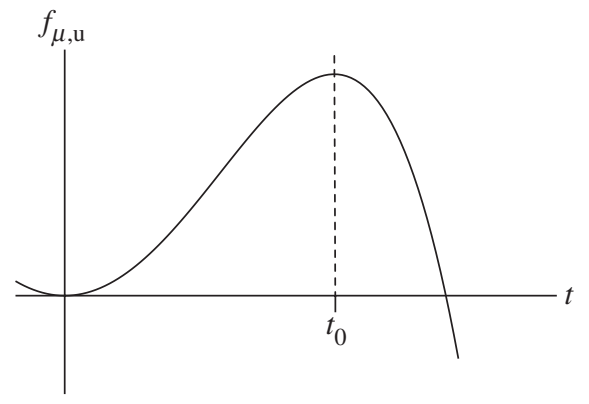

Figure 3. Definition of $t_{0}$.

\section{Assumption 3.4.}

(1) Assumption 2.9 holds.

(2) There exists a function $c(\mu)$ such that

$$
(2+\sigma) \Phi(u)-(2+\tau) \mu \Psi(u) \leqslant c(\mu)\|u\|^{2}, \quad \forall u \in H^{1},
$$

where

(i) $c(\mu)>0, \forall \mu>0$;

(ii) $\lim _{\mu \rightarrow 0^{+}} c(\mu)=+\infty$ and $\lim _{\mu \rightarrow+\infty} c(\mu)=0$;

(iii) $c(\cdot)$ is non-increasing on $(0,+\infty)$.

We have the following result.

Theorem 3.5. Suppose that assumption 3.4 holds. Then problem 2.5 has no solution $(\lambda, u) \in \mathbb{R} \times\left(H^{1} \backslash\{0\}\right)$ with $\lambda<\lambda_{1}-c(\mu)$ (see figure 2). Thus, a necessary condition for the existence of solutions is

$$
\lambda \geqslant \lambda_{1}-c(\mu) .
$$

Proof. By assumption 3.4 we have

$$
\begin{aligned}
J_{\lambda}^{\prime}(u) u & =B(u)-\lambda\|u\|^{2}-(2+\sigma) \Phi(u)+\mu(2+\tau) \Psi(u) \\
& \geqslant\left(\lambda_{1}-\lambda\right)\|u\|^{2}-c(\mu)\|u\|^{2} \\
& =\left[\lambda_{1}-c(\mu)-\lambda\right]\|u\|^{2} .
\end{aligned}
$$

Thus, $J_{\lambda}^{\prime}(u)=0$ implies that $\lambda_{1}-c(\mu)-\lambda \leqslant 0$.

\section{The critical sets $\mathfrak{M}_{\lambda}$ and $\mathfrak{N}_{\lambda}$}

We denote by

$$
\Omega:=\left\{u \in H^{1} \mid\|u\|_{1}=1\right\}
$$

the unit sphere in $H^{1}$ and we consider, for $u \in \Omega, \lambda \in \mathbb{R}$ and $\mu \in \mathbb{R}^{+}$fixed, the function

$$
s_{\lambda, \mu, u}(t):=J_{\lambda}(t u)=\frac{1}{2} B_{\lambda}(u) t^{2}-\Phi(u)|t|^{2+\sigma}+\mu \Psi(u)|t|^{2+\tau}, \quad t \in \mathbb{R},
$$




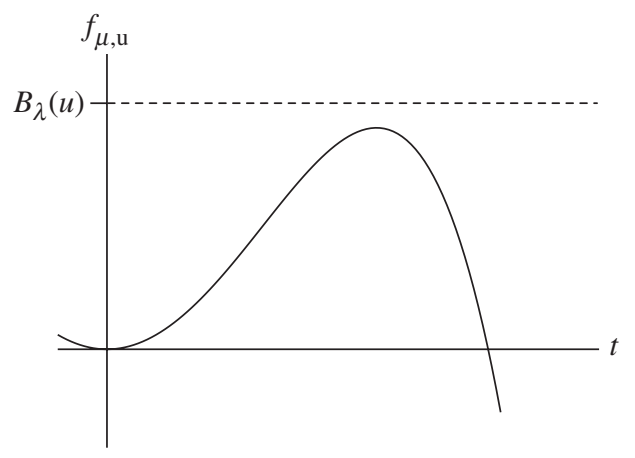

Figure 4. Situation where $u \in \Omega \backslash \mathfrak{K}_{\lambda}$.

with

$$
s_{\lambda, \mu, u}^{\prime}(t)=t\left[B_{\lambda}(u)-(2+\sigma) \Phi(u)|t|^{\sigma}+(2+\tau) \mu \Psi(u)|t|^{\tau}\right] .
$$

We set

$$
f_{\mu, u}(t):=(2+\sigma) \Phi(u)|t|^{\sigma}-(2+\tau) \mu \Psi(u)|t|^{\tau}, \quad t \in \mathbb{R},
$$

and we define $t_{0}(u)>0$ by the relation (see figure 3 )

$$
f_{\mu, u}\left(t_{0}(u)\right)=\max _{t>0} f_{\mu, u}(t) .
$$

Then

$$
s_{\lambda, \mu, u}^{\prime}(t)=0 \Longleftrightarrow\left\{\begin{array}{l}
t=0, \\
f_{\mu, u}(t)=B_{\lambda}(u) .
\end{array}\right.
$$

This property explains our interest in the two following subsets of $\Omega$ :

(i) $\mathfrak{K}_{\lambda}:=\left\{u \in \Omega \mid f_{\mu, u}\left(t_{0}(u)\right) \geqslant B_{\lambda}(u)\right\}$; note that $\Omega \cap E^{-} \subset \mathfrak{K}_{\lambda}$.

(ii) $\mathfrak{K}_{\lambda}^{+}:=\left\{u \in \mathfrak{K}_{\lambda} \mid B_{\lambda}(u)>0\right\}$.

For $u \in \mathfrak{K}_{\lambda}^{+}$, we define $t_{1}(u)$ by the relations

$$
t_{1}(u)>0, \quad f_{\mu, u}\left(t_{1}(u)\right)=B_{\lambda}(u) \quad \text { and } \quad f_{\mu, u}^{\prime}\left(t_{1}(u)\right) \geqslant 0 .
$$

For $u \in \mathfrak{K}_{\lambda}$, we define $t_{2}(u)$ by the relations

$$
t_{2}(u)>0, \quad f_{\mu, u}\left(t_{2}(u)\right)=B_{\lambda}(u) \quad \text { and } \quad f_{\mu, u}^{\prime}\left(t_{2}(u)\right) \leqslant 0 .
$$

\section{REMARK 4.1.}

(i) For $u \in \Omega \backslash \mathfrak{K}_{\lambda}$, we have the situation shown in figure 4 .

(ii) For $u \in \mathfrak{K}_{\lambda}^{+}$, we have either of the situations in figure 5 .

(iii) For $u \in \mathfrak{K}_{\lambda} \backslash \mathfrak{K}_{\lambda}^{+}, t_{1}(u)$ does not exist and we have the situation in figure 6

We consider now the mappings

$$
\kappa_{1}: \mathfrak{K}_{\lambda}^{+} \rightarrow H^{1}, \quad u \mapsto t_{1}(u) u,
$$



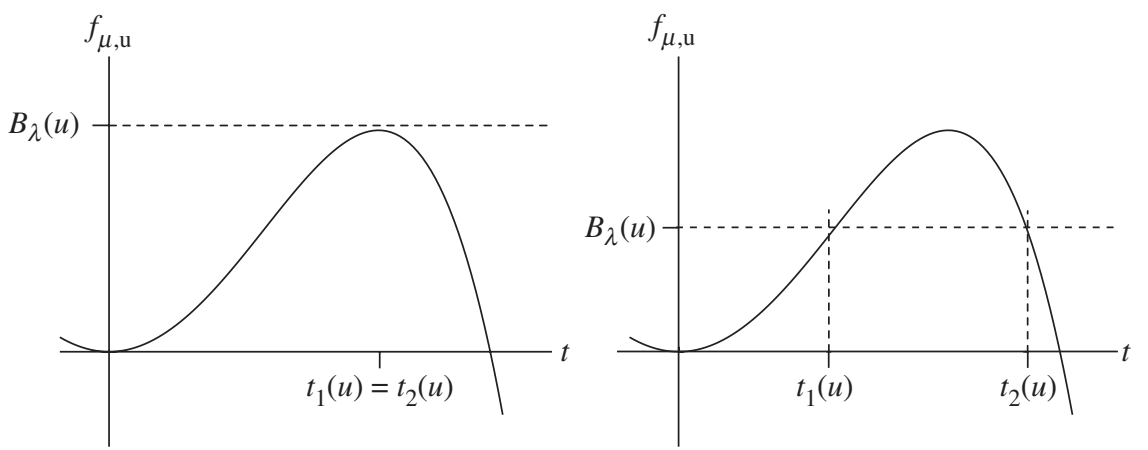

Figure 5. Situation where $u \in \mathfrak{K}_{\lambda}^{+}$.

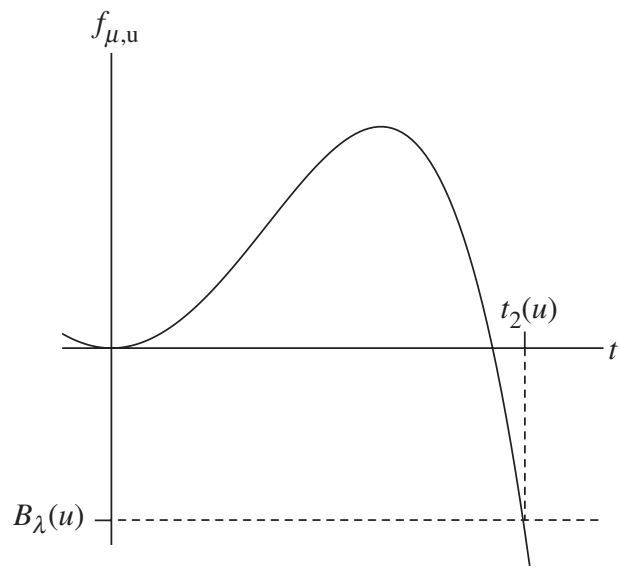

Figure 6. Situation where $u \in \mathfrak{K}_{\lambda} \backslash \mathfrak{K}_{\lambda}^{+}$.

and

$$
\kappa_{2}: \mathfrak{K}_{\lambda} \rightarrow H^{1}, \quad u \mapsto t_{2}(u) u,
$$

and we define the sets

$$
\mathfrak{M}_{\lambda}:=\kappa_{1}\left(\mathfrak{K}_{\lambda}^{+}\right) \quad \text { and } \quad \mathfrak{N}_{\lambda}:=\kappa_{2}\left(\mathfrak{K}_{\lambda}\right) .
$$

As soon as $\mathfrak{K}_{\lambda}^{+}=\emptyset$, we set $\mathfrak{M}_{\lambda}=\emptyset$; and, similarly, as soon as $\mathfrak{K}_{\lambda}=\emptyset$, we set $\mathfrak{N}_{\lambda}=\emptyset$. We remark, however, that $\mathfrak{N}_{\lambda} \neq \emptyset$ whenever $\lambda>\lambda_{1}$.

The following proposition summarizes our interest in these sets.

Proposition 4.2. Suppose that assumption 2.9 is correct. The equation $J_{\lambda}^{\prime}(u)=0$ then implies that

$$
u \in \mathfrak{N}_{\lambda} \cup \mathfrak{M}_{\lambda} \cup\{0\} .
$$

REMARK 4.3. Note that $\inf _{u \in \mathfrak{M}_{\lambda}} J_{\lambda}(u) \geqslant 0$ if $\mathfrak{M}_{\lambda} \neq \emptyset$. 


\section{A first solution set}

Our aim in this section is to establish the existence of a set of solutions $\left(\lambda, u_{1, \lambda}\right)$ for $\lambda<\lambda_{1}$. To this end we will use minimization on the set $\mathfrak{N}_{\lambda}$ as well as an associated mountain-pass theorem.

Proposition 5.1. Suppose that assumptions 2.9 and 3.4 hold, and suppose that $\lambda<\lambda_{1}$. There then exists a function $\mu_{1}(\lambda)$ such that

(i) $\mathfrak{N}_{\lambda} \neq \emptyset$ for $0<\mu<\mu_{1}(\lambda)$;

(ii) $\mathfrak{N}_{\lambda}=\emptyset$ for $\mu>\mu_{1}(\lambda)$.

The function $\mu_{1}(\lambda)$ is non-decreasing and satisfies the relation $\lambda \geqslant \lambda_{1}-c\left(\mu_{1}(\lambda)\right)$.

Proof. We show first that $\mathfrak{N}_{\lambda} \neq \emptyset$ as soon as $\mu>0$ is sufficiently small. Indeed, let us choose any $u \in \Omega$ and then fix any value of $t>0$ so that

$$
\frac{(2+\sigma) \Phi(t u)}{t^{2}} \geqslant \frac{3}{2} B_{\lambda}(u) .
$$

Such a choice of $t$ is possible, since $\Phi(t u) / t^{2}=t^{\sigma} \Phi(u)$ is a strongly increasing, positive function of $t$. Then we have, for sufficiently small $\mu>0$,

$$
\frac{(2+\sigma) \Phi(t u)}{t^{2}}-\mu \frac{(2+\tau) \Psi(t u)}{t^{2}}=f_{\mu, u}(t)>B_{\lambda}(u) .
$$

But this means that $\kappa_{2}(u) \in \mathfrak{N}_{\lambda}$ for such values of $\mu$.

Next we remark that as soon as $\mathfrak{N}_{\lambda} \neq \emptyset$ for some $\bar{\mu}$, we have a fortiori $\mathfrak{N}_{\lambda} \neq \emptyset$ for $\mu \in(0, \bar{\mu}]$.

The result follows now from the fact that $\mathfrak{N}_{\lambda}=\emptyset$ for $\mu$ sufficiently large (see theorem 3.5).

REMARK 5.2. When $\lambda<\lambda_{1}, \mathfrak{N}_{\lambda} \neq \emptyset$ if and only if $\mathfrak{M}_{\lambda} \neq \emptyset$.

Now, for $\lambda<\lambda_{1}$ and $\mu \in\left(0, \mu_{1}(\lambda)\right]$, we set

$$
m_{1}(\lambda):=\inf _{u \in \mathfrak{M}_{\lambda}} J_{\lambda}(u) \quad \text { and } \quad n_{1}(\lambda):=\inf _{u \in \mathfrak{N}_{\lambda}} J_{\lambda}(u)
$$

Clearly, $+\infty>m_{1}(\lambda) \geqslant n_{1}(\lambda) \geqslant-\infty$. We are interested in the finiteness of these quantities. In fact we put forward the following proposition.

Proposition 5.3. Suppose that assumptions 2.9 and 3.4 hold, that $\lambda<\lambda_{1}$ and that $0<\mu \leqslant \mu_{1}(\lambda)$. Then $+\infty>m_{1}(\lambda) \geqslant n_{1}(\lambda)>-\infty$.

Proof. Suppose, on the contrary, that there exists a sequence $\left\{u_{n}\right\} \subset \mathfrak{N}_{\lambda}$ such that $J_{\lambda}\left(u_{n}\right) \rightarrow-\infty$ as $n \rightarrow+\infty$. Since $J^{\prime}\left(u_{n}\right) u_{n}=0$, a computation similar to that leading to equation (3.2) gives

$$
\mu \Psi\left(u_{n}\right)=\frac{\sigma}{2(\tau-\sigma)} B_{\lambda}\left(u_{n}\right)-\frac{2+\sigma}{\tau-\sigma} J_{\lambda}\left(u_{n}\right) .
$$


If, up to some subsequence, we have $B_{\lambda}\left(u_{n}\right) \rightarrow+\infty$ for $n \rightarrow+\infty$, we may obtain $\mu \Psi\left(u_{n}\right) \rightarrow+\infty$; this would lead us to the contradiction

$$
J_{\lambda}\left(u_{n}\right) \geqslant-\Psi\left(u_{n}\right)\left[\frac{\Phi\left(u_{n}\right)}{\Psi\left(u_{n}\right)}-\mu\right] \rightarrow+\infty .
$$

If, up to some subsequence, we have that $B_{\lambda}\left(u_{n}\right)$ remains bounded, this same argument would lead us to the same contradiction.

Therefore, up to some subsequence, we should have $B_{\lambda}\left(u_{n}\right) \rightarrow-\infty$, contradicting $\lambda<\lambda_{1}$, and so we obtain the desired conclusion.

REMARK 5.4. For $\lambda>\lambda_{1}$, we have $\mathfrak{N}_{\lambda} \neq \emptyset$; in this case, the above argument no longer holds. It seems difficult to show that $n_{1}(\lambda)>-\infty$ holds for $\lambda>\lambda_{1}$ in the general case. With additional assumptions, this can be shown. For example if, whenever $B_{\lambda}\left(u_{n}\right) \rightarrow-\infty$, we have

$$
\mu \Psi\left(u_{n}\right) \rightarrow+\infty \text { and } B_{\lambda}\left(u_{n}\right) \geqslant-\operatorname{const} \Psi\left(u_{n}\right)^{2 /(2+\tau)},
$$

then we can proceed with the above argument for $B_{\lambda}\left(u_{n}\right) \rightarrow-\infty$ by showing that $J\left(u_{n}\right)$ must remain bounded from below. Indeed

$$
\begin{aligned}
J_{\lambda}\left(u_{n}\right) & =\frac{1}{2} B_{\lambda}\left(u_{n}\right)+\mu \Psi\left(u_{n}\right)(1+o(1)) \\
& \geqslant- \text { const. } \Psi\left(u_{n}\right)^{2 /(2+\tau)}+\mu \Psi\left(u_{n}\right)(1+o(1)) .
\end{aligned}
$$

Proposition 5.5. Suppose that assumptions 2.9 and 3.4 hold, that $\lambda<\lambda_{1}$ and that $0<\mu \leqslant \mu_{1}(\lambda)$. Then $m_{1}(\lambda)>0$.

Proof. We have, for $\|u\|_{1}$ sufficiently small, say $\|u\|_{1}=\rho>0$,

$$
\begin{aligned}
J_{\lambda}(u) & \geqslant I_{\lambda}(u)=\frac{1}{2} B_{\lambda}(u)-\Phi(u) \\
& \geqslant \frac{1}{2} \min \left\{1, \frac{\lambda_{1}-\lambda}{\lambda_{1}}\right\}\|u\|_{1}^{2}-\Phi(u) \quad \text { (by proposition 2.15) } \\
& =\|u\|_{1}^{2}\left\{\frac{1}{2} \min \left\{1, \frac{\lambda_{1}-\lambda}{\lambda_{1}}\right\}-\frac{\Phi(u)}{\|u\|_{1}^{2}}\right\} \\
& \geqslant \frac{1}{4} \min \left\{1, \frac{\lambda_{1}-\lambda}{\lambda_{1}}\right\}\|u\|_{1}^{2} \quad \text { (by proposition 2.11). }
\end{aligned}
$$

Thus, $m_{1}(\lambda) \geqslant \frac{1}{4} \min \left\{1,\left(\lambda_{1}-\lambda\right) / \lambda_{1}\right\} \rho^{2}>0$.

We will need the fact that $m_{1}(\lambda)>n_{1}(\lambda)$. This is so, for example, as soon as $n_{1}(\lambda)<0$. More generally, we have the following result.

Proposition 5.6. Suppose that assumptions 2.9 and 3.4 hold and that $\lambda<\lambda_{1}$. There then exists a function $\bar{\mu}_{1}(\lambda)$ with $\bar{\mu}_{1}(\lambda) \in\left(0, \mu_{1}(\lambda)\right]$ such that

$$
m_{1}(\lambda)>n_{1}(\lambda) \quad \text { for } \mu \in\left(0, \bar{\mu}_{1}(\lambda)\right] \text {. }
$$

Moreover, the function $\bar{\mu}_{1}(\cdot)$ can be chosen to be non-decreasing.

REMARK 5.7. We insist on the fact that we do not exclude the possibility that $\lim _{\lambda \rightarrow \lambda_{1}^{-}} \bar{\mu}_{1}(\lambda) \in \mathbb{R}$. 
Proof. It is enough to show that $n_{1}(\lambda)<0$ as soon as $\mu>0$ is sufficiently small. In order to achieve this, we fix any $u \in \Omega$ and choose $t>0$ in such a way that $\Phi(t u) / t^{2} \geqslant B_{\lambda}(u)$. Then

$$
J_{\lambda}(t u)=t^{2}\left[\frac{1}{2} B_{\lambda}(u)-\frac{\Phi(t u)}{t^{2}}+\mu \frac{\Psi(t u)}{t^{2}}\right]<-\frac{1}{4} t^{2} B_{\lambda}(u)<0
$$

for $\mu>0$ sufficiently small.

Proposition 5.8. Suppose that assumptions 2.9 and 3.4 hold, that $\lambda<\lambda_{1}$ and that $\mu \in\left(0, \bar{\mu}_{1}(\lambda)\right)$. Then the set $\mathfrak{M}_{\lambda} \cup \mathfrak{N}_{\lambda}$ is bounded away from zero.

Proof. Suppose, on the contrary, that we could find a sequence $\left\{u_{n}\right\}$ in $\mathfrak{M}_{\lambda} \cup \mathfrak{N}_{\lambda}$ with $\left\|u_{n}\right\|_{1} \rightarrow 0$. Then

$$
B_{\lambda}\left(u_{n}\right)=(2+\sigma) \Phi\left(u_{n}\right)-\mu(2+\tau) \Psi\left(u_{n}\right) \leqslant(2+\sigma) \Phi\left(u_{n}\right),
$$

so we may obtain, by propositions 2.15 and 2.11 , the contradiction

$$
\min \left\{1, \frac{\lambda_{1}-\lambda}{\lambda_{1}}\right\} \leqslant(2+\sigma) \frac{\Phi\left(u_{n}\right)}{\left\|u_{n}\right\|_{1}^{2}} \rightarrow 0 \quad \text { as } n \rightarrow+\infty
$$

Now everything is ready to apply variational methods, except that we need a manifold structure for a subset of $\mathfrak{N}_{\lambda}$ that does not intersect the set $\mathfrak{M}_{\lambda}$. Thus, we introduce, for $\lambda<\lambda_{1}$ and $\mu \in\left(0, \bar{\mu}_{1}(\lambda)\right)$, the set

$$
\mathfrak{N}_{\lambda, \varepsilon}:=\left\{u \in \mathfrak{N}_{\lambda} \mid J_{\lambda}(u) \leqslant m_{1}(\lambda)-\varepsilon\right\},
$$

where $\varepsilon \in\left(0, m_{1}(\lambda)-n_{1}(\lambda)\right)$.

Proposition 5.9. Suppose that assumptions 2.9 and 3.4 hold, that $\lambda<\lambda_{1}$ and that $\mu \in\left(0, \bar{\mu}_{1}(\lambda)\right)$. Then

(i) $\mathfrak{N}_{\lambda, \varepsilon}$ is a complete $C^{1}$-manifold (with boundary $\partial \mathfrak{N}_{\lambda, \varepsilon}$ ) of codimension 1 ;

(ii) the space $\mathfrak{N}_{\lambda, \varepsilon}$ intersects the ray $\operatorname{span}\{u\}$ transversally, i.e.

$$
\operatorname{span}\left\{T_{u} \mathfrak{N}_{\lambda, \varepsilon} \cup \operatorname{span}\{u\}\right\}=T_{u} H^{1}, \quad \forall u \in \mathfrak{N}_{\lambda, \varepsilon} \backslash \partial \mathfrak{N}_{\lambda, \varepsilon} .
$$

Proof. We set $h(u):=B_{\lambda}(u)-(2+\sigma) \Phi(u)+\mu(2+\tau) \Psi(u)=J_{\lambda}^{\prime}(u) u$ and we remark that

$$
h(u)=0 \Longleftrightarrow u \in \mathfrak{M}_{\lambda} \cup \mathfrak{N}_{\lambda} \cup\{0\} .
$$

We recall that, for $t>0$,

$$
\begin{aligned}
& s_{\lambda, \mu, u}^{\prime}(t)=B_{\lambda}(u) t-\frac{(2+\sigma) \Phi(t u)}{t}+\frac{(2+\tau) \Psi(t u)}{t} \\
& s_{\lambda, \mu, u}^{\prime \prime}(t)=B_{\lambda}(u)-\frac{(2+\sigma)(1+\sigma) \Phi(t u)}{t^{2}}+\frac{(2+\tau)(1+\tau) \Psi(t u)}{t^{2}},
\end{aligned}
$$


so that

$$
\begin{aligned}
s_{\lambda, \mu, u}^{\prime}(1) & =s_{\lambda, \mu, u}^{\prime \prime}(1)=0, \\
& \Longleftrightarrow\left\{\begin{array}{l}
B_{\lambda}(u)=(2+\sigma) \Phi(u)-(2+\tau) \Psi(u), \\
B_{\lambda}(u)=(2+\sigma)(1+\sigma) \Phi(u)-(2+\tau)(1+\tau) \Psi(u),
\end{array}\right. \\
& \Longleftrightarrow\left\{\begin{array}{l}
B_{\lambda}(u)=(2+\sigma) \Phi(u)-(2+\tau) \Psi(u), \\
2 B_{\lambda}(u)=(2+\sigma)^{2} \Phi(u)-(2+\tau)^{2} \Psi(u),
\end{array}\right. \\
& \Longleftrightarrow\left\{\begin{array}{l}
h(u)=0, \\
h^{\prime}(u) u=0 .
\end{array}\right.
\end{aligned}
$$

Hence,

$$
\left.\begin{array}{r}
h(u)=0 \\
h^{\prime}(u) u=0
\end{array}\right\} \Longleftrightarrow u \in\left(\mathfrak{M}_{\lambda} \cap \mathfrak{N}_{\lambda}\right) \cup\{0\} .
$$

Let us consider now some point $u \in \mathfrak{N}_{\lambda, \varepsilon}$. Then $h(u)=0$, but $h^{\prime}(u) u \neq 0$, since $u \notin \mathfrak{M}_{\lambda} \cup\{0\}$. By the inverse function theorem we find that

(i) $\mathfrak{N}_{\lambda, \varepsilon}$ is a $C^{1}$-manifold of codimension 1 in a neighbourhood of $u$;

(ii) $T_{u} \mathfrak{N}_{\lambda}=T_{u} \mathfrak{N}_{\lambda, \varepsilon}=\operatorname{ker} h^{\prime}(u)$.

So it remains to show that the manifold $\mathfrak{N}_{\lambda, \varepsilon}$ is closed. To this end, we consider a sequence $\left\{u_{n}\right\} \subset \mathfrak{N}_{\lambda, \varepsilon}$ with $u_{n} \rightarrow u$ as $n \rightarrow+\infty$. Then $h\left(u_{n}\right)=0$ implies that $h(u)=0$, so that $u \in \mathfrak{M}_{\lambda} \cup \mathfrak{N}_{\lambda} \cup\{0\}$. Since $\mathfrak{M}_{\lambda} \cup \mathfrak{N}_{\lambda}$ is bounded away from 0 , we necessarily have $u \in \mathfrak{M}_{\lambda} \cup \mathfrak{N}_{\lambda}$, and $J_{\lambda}\left(u_{n}\right) \leqslant m_{1}(\lambda)-\varepsilon$, i.e. $J_{\lambda}(u) \leqslant m_{1}(\lambda)-\varepsilon$ shows that $u \in \mathfrak{N}_{\lambda, \varepsilon}$.

THEOREM 5.10. Suppose that assumptions 2.9 and 3.4 hold. Suppose also that $\lambda<$ $\lambda_{1}$ and $\mu \in\left(0, \bar{\mu}_{1}(\lambda)\right)$.

There then exists an element $w_{1, \lambda} \in \mathfrak{N}_{\lambda}$ such that $J_{\lambda}\left(w_{1, \lambda}\right)=n_{1}(\lambda), J_{\lambda}^{\prime}\left(w_{1, \lambda}\right)=0$ and $w_{1, \lambda} \neq 0$. Hence $\left(\lambda, w_{1, \lambda}\right)$ is a solution of problem 2.5.

Proof. Consider a minimizing sequence $\left\{u_{n}\right\} \subset \mathfrak{N}_{\lambda}: J_{\lambda}\left(u_{n}\right) \rightarrow n_{1}(\lambda)$ for $n \rightarrow+\infty$. We choose a sequence of positive numbers $\left\{\varepsilon_{n}\right\}$ such that

$$
J_{\lambda}\left(u_{n}\right) \leqslant n_{1}(\lambda)+\varepsilon_{n}^{2}, \quad \varepsilon_{n} \rightarrow 0 .
$$

Without loss of generality we may assume that

$$
n_{1}(\lambda)+\varepsilon_{n}^{2}<m_{1}(\lambda) .
$$

We may now apply Ekeland's variational principle (see, for example, [22]) in order to obtain a new sequence $\left\{v_{n}\right\} \subset \mathfrak{N}_{\lambda}$ satisfying

$$
\begin{aligned}
J_{\lambda}\left(v_{n}\right) & \leqslant J_{\lambda}\left(v_{n}+w\right)+\varepsilon_{n}\|w\|_{1}, \quad \forall v_{n}+w \in \mathfrak{N}_{\lambda}, \\
J_{\lambda}\left(v_{n}\right) & \leqslant J_{\lambda}\left(u_{n}\right), \\
\left\|v_{n}-u_{n}\right\| & \leqslant \varepsilon_{n} .
\end{aligned}
$$


Now

$$
\left\|J_{\lambda}^{\prime}\left(v_{n}\right)\right\|_{-1}=\sup _{\substack{w \in T_{v_{n}} \mathfrak{N}_{\lambda} \\\|w\|_{1}=1}} J_{\lambda}^{\prime}\left(v_{n}\right) w
$$

So

$$
J_{\lambda}\left(v_{n}+w\right)-J_{\lambda}\left(v_{n}\right) \geqslant-\varepsilon_{n}\|w\|_{1}, \quad \forall v_{n}+w \in \mathfrak{N}_{\lambda}
$$

implies that $J_{\lambda}^{\prime}\left(v_{n}\right) w \geqslant-\varepsilon_{n}\|w\|_{1}$, such that (replacing $w$ by $-w$ ) we obtain

$$
\left\|J_{\lambda}^{\prime}\left(v_{n}\right)\right\|_{-1} \leqslant \varepsilon_{n} .
$$

By the Palais-Smale condition established above we may now assume that, up to some subsequence, we have $v_{n} \rightarrow v$ in $H^{1}$. Thus, we obtain

$$
v \in \mathfrak{N}_{\lambda}, \quad J_{\lambda}^{\prime}(v)=0, \quad J_{\lambda}(v)=n_{1}(\lambda) .
$$

We call this element $w_{1, \lambda}$ !

REMARK 5.11. If $J_{\lambda}$ is an even function, we in fact obtain a solution pair $\left(\lambda, \pm w_{1, \lambda}\right)$.

REMARK 5.12. In the context given in remark 5.4, the solution $\left(\lambda, w_{1, \lambda}\right)$ corresponding to the critical value $n_{1}(\lambda)$ exists for $\left.\lambda \in\right] \lambda_{1}, U\left[\backslash \sigma_{p}\left(L_{1}\right)\right.$, too, since the above proof remains valid in this case, this being so for all values of $\mu>0$.

The next theorem gives us the existence of a second critical point, independent of any additional symmetry assumption on $J_{\lambda}(\cdot)$.

TheOrem 5.13. Suppose that assumptions 2.9 and 3.4 hold. Suppose also that $\lambda<$ $\lambda_{1}$ and $\mu \in\left(0, \bar{\mu}_{1}(\lambda)\right)$.

There then exists a second critical point $v_{1, \lambda}$ of $J_{\lambda}$ such that

$$
J_{\lambda}\left(v_{1, \lambda}\right) \geqslant m_{1}(\lambda)>n_{1}(\lambda), \quad J_{\lambda}^{\prime}\left(v_{1, \lambda}\right)=0, \quad v_{1, \lambda} \notin\left\{w_{1, \lambda}, 0\right\} .
$$

Proof. We consider a set of paths

$$
\mathcal{P}:=\left\{p \in C^{0}([0,1] ; h) \mid p(0)=w_{1, \lambda}, p(1)=0\right\} .
$$

We then consider

$$
\bar{m}_{1}(\lambda):=\inf _{p \in \mathcal{P}} \sup _{u \in p} J_{\lambda}(u) .
$$

We remark that $\bar{m}_{1}(\lambda) \geqslant m_{1}(\lambda)>\max \left\{0, n_{1}(\lambda)\right\}$.

Now a classical mountain-pass argument gives the desired result.

REMARK 5.14. If $J_{\lambda}$ is an even function, we in fact obtain a solution pair $\left(\lambda, \pm v_{1, \lambda}\right)$.

\section{The existence of other solutions}

In $\S 5$ we established the existence of two 'non-trivial' critical values $n_{1}(\lambda)$ and $\bar{m}_{1}(\lambda)$ of $J_{\lambda}(u)$ for $\lambda<\lambda_{1}$, as long as $\mu>0$ is sufficiently small, i.e. as long as

$$
0<\mu<\bar{\mu}_{1}(\lambda)
$$

where $\bar{\mu}_{1}(\lambda)$ is a non-decreasing function defined on $\left(-\infty, \lambda_{1}\right)$. Moreover, we have seen that there are no 'non-trivial' critical values if $\mu>0$ is sufficiently large. 
When $\lambda>\lambda_{1}$, say $\lambda \in\left(\lambda_{1}, \lambda_{2}\right)$, for example, $\mathfrak{N}_{\lambda} \neq \emptyset$ even if $\mu>0$ is large, but there is no evidence for $n_{1}(\lambda)$ to be finite in general, this being so even if $\mu>0$ is small (see, however, remark 5.4). This new situation calls for a new approach when studying the existence of solutions of problem 2.5 for $\lambda>\lambda_{1}$. It will turn out that this new variational characterization will lead us to new critical values even if $\lambda<\lambda_{1}$.

But before presenting this new approach, let us introduce a somewhat stronger assumption.

\section{Assumption 6.1.}

(i) Assumption 2.9 is fulfilled.

(ii) $\operatorname{dim} H^{1}=+\infty$.

(iii) The functionals $\Phi$ and $\Psi$ are even and belong to $C^{2}$.

(iv) The eigenspace $E\left(\lambda_{i}\right)$ corresponding to $L_{1}-\lambda_{i}$ is of finite dimension for $i=1, \ldots, \bar{M}$, where $\bar{M} \leqslant M$.

Suppose now that

$$
\lambda \in\left(-\infty, \lambda_{k_{0}}\right) \backslash \sigma_{p}\left(L_{1}\right) \text { for some } k_{0} \in\{1, \ldots, \bar{M}+1\}
$$

and let

$$
d\left(k_{0}\right):=\sum_{i=1}^{k_{0}} \operatorname{dim} E\left(\lambda_{i}\right) \quad \text { if } k_{0} \neq \bar{M}+1 \text { and } d(\bar{M}+1)=+\infty ;
$$

for convenience we set $d(0)=0$. We consider the set

$$
\mathfrak{A}:=\left\{A \subset \mathfrak{N}_{\lambda} \mid A \text { is compact and } A=-A\right\}
$$

and we denote by $\gamma(\cdot)$ the Krasnoselski genus of symmetric sets in $\mathfrak{A}$. For $k=$ $1, \ldots, d\left(k_{0}\right)$, we introduce the sets

$$
\mathfrak{F}_{k}:=\{A \in \mathfrak{A} \mid \gamma(A) \geqslant k\}
$$

and we look at the following candidates for critical values

$$
n_{k}(\lambda):=\inf _{A \in \mathfrak{F}_{k}} \sup _{u \in A} J_{\lambda}(u),
$$

with $n_{k}(\lambda)=+\infty$ if $\mathfrak{F}_{k}=\emptyset$. Note that, for $\lambda<\lambda_{1}$ and $k=1$, this definition coincides with the former definition of $n_{1}(\lambda)$ used in the previous section.

Our first interest lies in the finiteness of $n_{k}(\lambda)$.

Proposition 6.2. Suppose that assumptions 2.9, 3.4 and 6.1 are fulfilled and let $k_{0} \in\{1, \ldots, \bar{M}+1\}$. Then the following hold.

(1) For every $k \in\left\{d\left(k_{0}-1\right)+1, \ldots, d\left(k_{0}\right)\right\}$ (or $k>d\left(k_{0}-1\right)$ if $\left.d\left(k_{0}\right)=\infty\right)$, there exists a non-decreasing function $\mu_{k}(\lambda)$ defined on $\left(-\infty, \lambda_{k_{0}}\right)$, such that

(i) $\mathfrak{F}_{k} \neq \emptyset$ if $0<\mu<\mu_{k}(\lambda)$, 
(ii) $\mathfrak{F}_{k}=\emptyset$ if $\mu>\mu_{k}(\lambda)$.

Moreover, for $i=1, \ldots, k_{0}$,

$$
0<\mu_{k+1}(\lambda) \leqslant \mu_{k}(\lambda) \quad \text { on }\left(-\infty, \lambda_{i}\right), \quad \forall k \in\{d(i-1)+1, \ldots, d(i)\} .
$$

(2) For every $k \in\left\{d\left(k_{0}-1\right)+1, \ldots, d\left(k_{0}\right)\right\}$, we have $n_{k}(\lambda) \in \mathbb{R}$ for $\lambda \in\left(-\infty, \lambda_{k_{0}}\right)$ and $0<\mu<\mu_{k}(\lambda)$.

Before proceeding with the proof, we make a remark. Suppose, for example, that $\operatorname{dim} E\left(\lambda_{1}\right)=1, \operatorname{dim} E\left(\lambda_{2}\right)=2$ and $\bar{M}=M=2$; we then find that

(1) $\mu_{1}(\lambda)$ is defined on $\left(-\infty, \lambda_{1}\right)$ and

$$
n_{1}(\lambda) \in \mathbb{R} \quad \text { for } \lambda<\lambda_{1} \text { and } 0<\mu<\mu_{1}(\lambda) .
$$

Moreover,

$$
\mu_{1}(\lambda) \geqslant \mu_{2}(\lambda) \geqslant \mu_{3}(\lambda) \geqslant \cdots \quad \text { on }\left(-\infty, \lambda_{1}\right)
$$

(2) $\mu_{2}(\lambda)$ and $\mu_{3}(\lambda)$ are defined on $\left(-\infty, \lambda_{2}\right)$ and

$$
\begin{array}{ll}
n_{2}(\lambda) \in \mathbb{R} & \text { for } \lambda<\lambda_{2} \text { and } 0<\mu<\mu_{2}(\lambda), \\
n_{3}(\lambda) \in \mathbb{R} & \text { for } \lambda<\lambda_{2} \text { and } 0<\mu<\mu_{3}(\lambda) \leqslant \mu_{2}(\lambda) .
\end{array}
$$

Moreover,

$$
\mu_{2}(\lambda) \geqslant \mu_{3}(\lambda) \geqslant \mu_{4}(\lambda) \geqslant \cdots \quad \text { on }\left(-\infty, \lambda_{2}\right)
$$

(3) $\mu_{k}(\lambda)(k \geqslant 4)$ is defined on $\left(-\infty, \lambda_{3}\right)$ and

$$
n_{k}(\lambda) \in \mathbb{R} \quad \text { for } \lambda<\lambda_{3} \text { and } 0<\mu<\mu_{k}(\lambda) .
$$

Moreover,

$$
\mu_{4}(\lambda) \geqslant \mu_{5}(\lambda) \geqslant \mu_{6}(\lambda) \geqslant \cdots \quad \text { on }\left(-\infty, \lambda_{3}\right)
$$

Proof. We begin the proof by choosing a compact set $A \subset \Omega$ with $A=-A$ in such a way that $\gamma(A)=k$. Such a choice is always possible and there exists a $b \in \mathbb{R}$ such that

$$
\sup _{u \in A} B_{\lambda}(u)=b .
$$

We then determine some $t \geqslant 1$ such that

$$
(2+\sigma) \frac{\Phi(t u)}{t^{2}} \geqslant \frac{3}{2} b, \quad \forall u \in A .
$$

Such a $t$ exists, for otherwise we could find a sequence $\left\{u_{n}\right\} \subset A$ such that

$$
(2+\sigma) \frac{\Phi\left(n u_{n}\right)}{n^{2}}=n^{\sigma}(2+\sigma) \Phi\left(u_{n}\right)<\frac{3}{2} b .
$$

Thus, we would have that $\lim _{n \rightarrow+\infty} \Phi\left(u_{n}\right)=0$. But, $A$ being compact, and if necessary taking a subsequence, we could assume that $u_{n} \rightarrow u \in A$ as $n \rightarrow \infty$. By continuity, this would lead us to the contradiction

$$
\Phi(u)=0 \quad \text { for some } u \in \Omega \text {. }
$$


Since $A$ is compact, $\Psi(t u)$ is bounded on $u \in A$. Thus, we may choose $\mu>0$ to be sufficiently small (say $\mu<\mu_{k}(\lambda)$ ), such that

$$
(2+\sigma) \frac{\Phi(t u)}{t^{2}}-\mu(2+\tau) \frac{\Psi(t u)}{t^{2}} \geqslant \frac{5}{4} b, \quad \forall u \in A .
$$

Thus, we could show that

$$
f_{\mu, u}(t) \geqslant \frac{5}{4} b \geqslant \frac{5}{4} B_{\lambda}(u), \quad \forall u \in A,
$$

and thus that, in fact, $A \subset \mathfrak{K}_{\lambda}$.

By the inverse function theorem, $t_{2}(u)$ depends continuously on $u \in A$. Thus, $\gamma\left(\kappa_{2}(A)\right) \geqslant k$.

Note that if we increase $\lambda, b$ will be decreasing, so $\mu_{k}(\lambda)$ is non-decreasing.

All that remains to be shown is that $\mathfrak{F}_{k}=\emptyset$ as soon as $\mu>0$ is sufficiently large and $k_{0} \geqslant 2$. Suppose indeed that $\mu$ is so large that $\lambda_{k_{0}}-\lambda>c(\mu)$. Let $P: H^{1} \rightarrow \bigoplus_{i=1}^{k_{0}-1} E\left(\lambda_{i}\right)$ be the orthogonal projection. Then

$$
\begin{aligned}
J_{\lambda}^{\prime}(u) u & =B(u)-\lambda\|u\|^{2}-(2+\sigma) \Phi(u)+\mu(2+\tau) \Psi(u) \\
& \geqslant\left(\lambda_{k_{0}}-\lambda\right)\|u\|^{2}-c(\mu)\|u\|^{2} \\
& =\left(\lambda_{k_{0}}-\lambda-c(\mu)\right)\|u\|^{2}>0, \quad \forall u \text { with } P(u)=0 .
\end{aligned}
$$

But this means that whenever $A \in \mathfrak{F}_{k}$ we would have $\gamma(A) \leqslant d\left(k_{0}-1\right)$. Indeed, in this case we obtain

$$
P(A) \subset \bigoplus_{i=1}^{k_{0}-1} E\left(\lambda_{i}\right) \backslash\{0\} \cong \mathbb{R}^{d\left(k_{0}-1\right)} \backslash\{0\} .
$$

Finally, note that $\mathfrak{F}_{k+1} \subset \mathfrak{F}_{k}$, such that the first point is established.

Concerning the second point, note that $\mathfrak{F}_{k} \neq \emptyset$ implies that $n_{k}(\lambda)<+\infty$. Moreover, for any set $A$ in $\mathfrak{F}_{k}$ with $k>d\left(k_{0}-1\right)$, we have $0 \in P(A)$, where $P$ is the orthogonal projection $H^{1} \rightarrow \bigoplus_{i=1}^{k_{0}-1} E\left(\lambda_{i}\right)$ introduced above. Hence, there always exists an element $u_{A} \in A$ such that $u_{A} \in E_{\lambda}^{+}$. Suppose now for a moment that $n_{k}(\lambda)=-\infty$. We could then obtain a sequence $\left\{u_{A_{n}}\right\} \subset \mathfrak{N}_{\lambda}$ with

$$
J_{\lambda}\left(u_{A_{n}}\right) \rightarrow-\infty \text { and } J_{\lambda}^{\prime}\left(u_{A_{n}}\right) u_{A_{n}}=0 .
$$

The same argument as that in the proof of proposition 5.3 can now be used in order to find

$$
B_{\lambda}\left(u_{A_{n}}\right) \rightarrow-\infty \text { as } n \rightarrow+\infty,
$$

contradicting in this way the choice of $u_{A_{n}} \in E_{\lambda}^{+}$.

The above proposition ensures finiteness of $n_{k}(\lambda)$, but we need to know that $n_{k}(\lambda)$ is even negative.

Proposition 6.3. Assume that the assumptions 2.9, 3.4 and 6.1 hold and let $k_{0} \in$ $\{1, \ldots, \bar{M}+1\}$.

Then, for every $k \in\left\{d\left(k_{0}-1\right)+1, \ldots, d\left(k_{0}\right)\right\}\left(\right.$ or $k>d\left(k_{0}-1\right)$ if $\left.d\left(k_{0}\right)=\infty\right)$ there exists a non-decreasing function $\bar{\mu}_{k}(\lambda)$ defined on $\left(-\infty, \lambda_{k_{0}}\right)$, such that

(i) $0<\bar{\mu}_{k}(\lambda) \leqslant \mu_{k}(\lambda)$, where $\mu_{k}(\lambda)$ is given in proposition 6.2, 
(ii) $n_{k}(\lambda)<0$ for $\lambda \in\left(-\infty, \lambda_{k_{0}}\right)$ and $0<\mu<\bar{\mu}_{k}(\lambda)$.

Moreover, for $i=1, \ldots, k_{0}$,

$$
0<\bar{\mu}_{k+1}(\lambda) \leqslant \bar{\mu}_{k}(\lambda) \quad \text { on }\left(-\infty, \lambda_{i}\right), \quad \forall k \in\{d(i-1)+1, \ldots, d(i)\} .
$$

Proof. We choose a compact set $A \subset \Omega$ with $A=-A$ in such a way that $\gamma(A)=k$ and we set

$$
b:=\sup _{u \in A} B_{\lambda}(u) .
$$

Note that $b>0$, since $k \geqslant d\left(k_{0}-1\right)$. We then determine some $t>0$ such that

$$
\frac{\Phi(t u)}{t^{2}} \geqslant b, \quad \forall u \in A .
$$

Such a choice is possible, for otherwise we could find a sequence $\left\{u_{n}\right\} \subset A$ with $n^{\sigma} \Phi\left(u_{n}\right) \leqslant b$, i.e. $\Phi\left(u_{n}\right) \rightarrow 0$. $A$ being compact, we may assume, up to a subsequence, that $u_{n} \rightarrow u \in \Omega, \Phi(u)=0$, obtaining in this way a contradiction.

Since $A$ is compact, $\Psi(t u)$ is bounded for $u \in A$. Thus, we may choose $\mu>0$ sufficiently small (say $\mu<\bar{\mu}(\lambda)$ ), such that

$$
\Phi(t u)-\mu \Psi(t u) \geqslant \frac{3}{4} b t^{2}, \quad \forall u \in A .
$$

Thus,

$$
J_{\lambda}(t u) \leqslant \frac{1}{2} B_{\lambda}(u) t^{2}-\frac{3}{4} b t^{2} \leqslant-\frac{1}{4} b t^{2}, \quad \forall u \in A .
$$

So $A \subset \mathfrak{K}_{\lambda}$ and $\kappa_{2}(A) \subset \mathfrak{F}_{k}$ with

$$
\inf _{u \in \kappa_{2}(A)} J_{\lambda}(u)<0
$$

Finally, note that if we increase $\lambda, \bar{\mu}_{k}(\lambda)$ will not decrease.

We consider now, for $\varepsilon \in\left(0,-\frac{1}{2} n_{k}(\lambda)\right)$, the set

$$
\mathfrak{N}_{\lambda, \varepsilon}:=\left\{u \in \mathfrak{N}_{\lambda} \mid J_{\lambda}(u) \leqslant-\varepsilon\right\} .
$$

Proposition 6.4. Suppose that assumptions 2.9, 3.4 and 6.1 hold. Let

$$
k_{0} \in\{1, \ldots, \bar{M}+1\}
$$

and suppose that $\lambda \in\left(-\infty, \lambda_{k_{0}}\right)$ and $0<\mu<\bar{\mu}(\lambda)$, where $\bar{\mu}_{k}(\lambda)$ is given by proposition 6.3 .

Then $\mathfrak{N}_{\lambda, \varepsilon}$ is a complete, symmetric Finsler manifold (with boundary $\partial \mathfrak{N}_{\lambda, \varepsilon}$ ) of class $C^{1,1}$ and of codimension 1 . Moreover, $T_{u} \mathfrak{N}_{\lambda, \varepsilon}$ and $\operatorname{span} u$ intersect transversally, i.e.

$$
\operatorname{span}\left\{T_{u} \mathfrak{N}_{\lambda, \varepsilon} \cup \operatorname{span}\{u\}\right\}=T_{u} H^{1}, \quad \forall u \in \mathfrak{N}_{\lambda, \varepsilon} \backslash \partial \mathfrak{N}_{\lambda, \varepsilon} .
$$

Proof. The proof of proposition 5.9 can be carried over with obvious adaptations.

By a classical argument (see, for example, [22]) we find that $n_{k}(\lambda)$ is a critical value of $J_{\lambda}$. Moreover, if $n_{k-1}(\lambda)=n_{k}(\lambda)$, then we obtain infinitely many critical points at level $n_{k}(\lambda)$. Thus, we obtain the following important result. 
TheOREM 6.5. Suppose that the assumptions 2.9, 3.4 and 6.1 hold; let

$$
k_{0} \in\{1, \ldots, \bar{M}+1\} \text {. }
$$

Suppose that $\lambda \in\left(-\infty, \lambda_{k_{0}}\right) \backslash \sigma_{p}\left(L_{1}\right)$ is such that

$$
0<\mu<\bar{\mu}_{k}(\lambda) \text { for some } k>d\left(k_{0}-1\right) \text {. }
$$

Then $J_{\lambda}$ has (at least) $k-d\left(k_{0}-1\right)$ pairs of critical points

$$
\pm w_{i, \lambda}, \quad i=d\left(k_{0}-1\right)+1, \ldots, k .
$$

The corresponding critical values are all distinct from zero and

$$
J_{\lambda}\left( \pm w_{i, \lambda}\right)=n_{i}(\lambda) \quad \text { for } i=d\left(k_{0}-1\right)+1, \ldots, k .
$$

When one must verify the condition in the above equation (6.1), it is interesting to recall that, as soon as we have $0<\mu<\bar{\mu}_{k}(\bar{\lambda})$ for some $\bar{\lambda}$, we find that

$$
0<\mu<\bar{\mu}_{k}(\lambda), \quad \forall \lambda \in\left[\bar{\lambda}, \lambda_{k_{0}}\right)
$$

and the solutions $\pm w_{i, \lambda}$ exist for $\lambda \in\left[\bar{\lambda}, \lambda_{k_{0}}\right) \backslash \sigma_{p}\left(L_{1}\right)$. They continue to exist even for $\lambda \in\left[\bar{\lambda}, U\left[\backslash \sigma_{p}\left(L_{1}\right)\right.\right.$ in the context given by remark 5.4 , since $n_{k}(\lambda) \geqslant n_{1}(\lambda)$.

We recall now that, for $\lambda \in\left(-\infty, \lambda_{1}\right)$, we could associate with the critical value $n_{1}(\lambda)$ another critical value, $\bar{m}_{1}(\lambda)$. We will now show that this procedure can be extended to $n_{k}(\lambda)$.

A closer analysis of the proofs of propositions $6.2,6.3$ and 5.6 shows the following.

Proposition 6.6. Assume that assumptions 2.9, 3.4 and 6.1 hold and let $k_{0} \in$ $\{1, \ldots, \bar{M}+1\}$.

The following hold.

(1) For all $k \in\left\{d\left(k_{0}-1\right)+1, \ldots, d\left(k_{0}\right)\right\}$ (or $k>d\left(k_{0}-1\right)$ if $d\left(k_{0}\right)=\infty$ ) there exists a non-decreasing function $\bar{\mu}_{k}(\lambda)$ defined on $\left(-\infty, \lambda_{k_{0}}\right)$ such that

(i) $\mathfrak{F}_{k} \neq \emptyset$ if $0<\mu<\bar{\mu}_{k}(\lambda)$,

(ii) $\exists \tilde{A} \in \mathfrak{F}_{k}$ such that $\tilde{A}$ is contained in some finite-dimensional space (of dimension $k+1)$ and $\sup _{u \in \tilde{A}} J_{\lambda}(u)<0$.

Moreover, for $i=1, \ldots, k_{0}$,

$$
0<\bar{\mu}_{k+1}(\lambda) \leqslant \bar{\mu}_{k}(\lambda) \quad \text { on }\left(-\infty, \lambda_{i}\right), \quad \forall k \in\{d(i-1)+1, \ldots, d(i)\} .
$$

(2) For all $k \in\left\{d\left(k_{0}-1\right)+1, \ldots, d\left(k_{0}\right)\right\}$, we have

$$
n_{k}(\lambda) \in(-\infty, 0) \text {, }
$$

for $\lambda \in\left(-\infty, \lambda_{k_{0}}\right)$ and $0<\mu<\bar{\mu}_{k}(\lambda)$.

(3) For $\lambda<\lambda_{1}$, there exist $\rho>0$ and $c>0$ such that

$$
J_{\lambda}(u)>0 \text { on } B_{\rho}(0) \backslash\{0\} \quad \text { and } \quad J_{\lambda}(u) \geqslant c>0 \text { on } \partial B_{\rho}(0) .
$$

For $\lambda \in\left(\lambda_{i-1}, \lambda_{i}\right), i \in\left\{2, \ldots, k_{0}\right\}$, there exist $\rho>0$ and $c>0$ such that

$$
J_{\lambda}(u)>0 \text { on }\left(B_{\rho}(0) \backslash\{0\}\right) \cap E_{\lambda}^{+} \quad \text { and } \quad J_{\lambda}(u) \geqslant c>0 \text { on } \partial B_{\rho}(0) \cap E_{\lambda}^{+} \text {. }
$$


We follow now Ambrosetti and Rabinowitz [2] and set

$$
\begin{aligned}
& \Gamma:=\left\{h \in C\left(H^{1} ; H^{1}\right) \mid h\right. \text { is an odd homeomorphism } \\
& \text { of } \left.H^{1} \text { onto } H^{1} \text { with } h(B) \subset \hat{I}_{0} \cup \bar{B}_{\rho}(0)\right\},
\end{aligned}
$$

where $B:=B_{1}(0), \hat{I}_{0}=\left\{u \in H^{1} \mid J_{\lambda}(t u) \geqslant 0\right.$ for $\left.t \in[0,1]\right\}$ and $\rho>0$ is given in the above proposition. Note that $\bar{B}_{\rho}(0) \subset \hat{I}_{0}$, for $\lambda<\lambda_{1}$.

Under the assumptions of proposition 6.6 and for $k \in\left\{d(k-1)+1, \ldots, d\left(k_{0}\right)\right\}$, $\mu<\bar{\mu}_{k}(\lambda), \lambda \in\left(-\infty, \lambda_{k_{0}}\right) \backslash \sigma_{p}\left(L_{1}\right)$, we set

$$
\Gamma_{k}:=\left\{A \subset H^{1} \mid A \text { is compact, } A=-A \text { and } \gamma(A \cap h(S)) \geqslant k, \forall h \in \Gamma\right\},
$$

where $S=\partial B_{1}(0)$. We remark that $A:=\{t u \mid t \in[0,1], u \in \tilde{A}\} \in \Gamma_{k}$, where $\tilde{A} \in$ $\mathfrak{F}_{k}$ is given in proposition 6.6. Indeed, $h(B)$ is an open neighbourhood of 0 in $H^{1}$, so that $h(B) \cap \operatorname{span}\{\tilde{A}\}$ is a neighbourhood of 0 . But $h(B) \cap \operatorname{span}\{\tilde{A}\}=h(B) \cap A$ and

$$
u \in \partial(h(B) \cap A) \Longrightarrow\left\{\begin{array} { l } 
{ u \in A } \\
{ u \in \partial ( h ( B ) ) }
\end{array} \Longrightarrow \left\{\begin{array}{l}
u \in A \\
u \in h(S)
\end{array}\right.\right.
$$

imply that

$$
\gamma(A \cap h(S)) \geqslant \gamma(\partial(h(B) \cap A)) \geqslant k .
$$

We consider now

$$
m_{k}(\lambda):=\inf _{A \in \Gamma_{k}} \sup _{u \in A} J_{\lambda}(u) .
$$

Note that $m_{k}(\lambda) \geqslant c>0$. In order to see this, take $h(u)=\rho u$ and note that $\gamma(A \cap h(S)) \geqslant k$ implies, as we have seen above, that $A \cap h(S) \cap E_{\lambda}^{+} \neq \emptyset$, so that the claim follows.

Using a classical deformation argument (see [2]), we find that $m_{k}(\lambda)$ is a critical value, together with the 'classical' multiplicity result for $m_{k}(\lambda)=m_{k+1}(\lambda)$.

Hence, we obtain the following theorem.

Theorem 6.7. Suppose that the assumptions 2.9, 3.4 and 6.1 hold and let $k_{0} \in$ $\{1, \ldots, \bar{M}+1\}$. Suppose also that $\lambda \in\left(-\infty, \lambda_{k_{0}}\right) \backslash \sigma_{p}\left(L_{1}\right)$ is such that

$$
0<\mu<\bar{\mu}_{k}(\lambda) \text { for some } k>d\left(k_{0}-1\right) .
$$

Then $J_{\lambda}$ has (at least) $k-d(k-1)$ different pairs of critical pairs

$$
\pm v_{i, \lambda} \quad \text { and } \quad \pm w_{i, \lambda}, \quad i=d\left(k_{0}-1\right)+1, \ldots, k .
$$

The corresponding critical values are all distinct from zero and

$J_{\lambda}\left( \pm v_{i, \lambda}\right)=m_{i}(\lambda)>0 \quad$ and $\quad J_{\lambda}\left( \pm w_{i, \lambda}\right)=n_{i}(\lambda)<0 \quad$ for $i=d\left(k_{0}-1\right)+1, \ldots, k$.

We recall that, as soon as $0<\mu<\bar{\mu}_{k}(\lambda)$ for some $\lambda$, this inequality still holds if one makes $\lambda$ larger. Hence, we can speak of 'branches' of solutions

$$
\left(\lambda, \pm v_{i, \lambda}\right) \text { and }\left(\lambda, \pm w_{i, \lambda}\right)
$$

for $\lambda \in(a, b)$, where $a<b$ and $b \in\left\{\lambda_{1}, \ldots, \lambda_{\bar{M}+1}\right\}$. 


\section{A concrete problem}

We now apply the abstract setting developed above to the following concrete problem.

Problem 7.1. 1.1 Find $\lambda \in \mathbb{R}$ and $u \in H^{1}(\mathbb{R}) \backslash\{0\}$ such that

$$
-u^{\prime \prime}(x)+V(x) u(x)-q(x)|u(x)|^{\sigma} u(x)+\mu r(x)|u(x)|^{\tau} u(x)=\lambda u(x), \quad x \in \mathbb{R},
$$

holds (in a generalized sense).

We thereby make the following assumption.

Assumption 7.2. $q(\cdot)$ and $r(\cdot)$ are positive functions in $L^{\infty}(\mathbb{R})$ and $0<\sigma<\tau$. $\mu$ is a positive parameter that measures the strength of the second nonlinear term, where we assume that $|r(\cdot)|_{L^{\infty}}=1$. We suppose that the function $V(x)$ has the following shape:

$$
V(x)= \begin{cases}U_{1}, & x<0, \\ 0, & 0 \leqslant x \leqslant a, \\ U_{2}, & x>a,\end{cases}
$$

where we assume without loss of generality that $0<U_{1} \leqslant U_{2} ; a$ is some positive constant and we assume that

$$
a \sqrt{U_{1}} \geqslant \frac{1}{2} \pi-\arcsin \sqrt{\frac{U_{1}}{U_{2}}} .
$$

On setting $L u:=-u^{\prime \prime}+V u, \boldsymbol{D}(L)=H^{2}(\mathbb{R})$ and $H:=L^{2}(\mathbb{R})$, the following proposition is well known (see [14]).

Proposition 7.3. Under assumption 7.2, assumptions 2.1 and 2.2 are fulfilled:

(i) $L: \boldsymbol{D}(L) \rightarrow L^{2}(\mathbb{R})$ is self-adjoint,

(ii) there exists $\ell>0$ such that $(L u, u) \geqslant \ell\|u\|^{2}, \forall u \in \boldsymbol{D}(L)$, where $(\cdot, \cdot)$ is the usual scalar product in $L^{2}(\mathbb{R})$ and $\|\cdot\|$ is the associated norm in $L^{2}(\mathbb{R})$,

(iii) the spectrum $\sigma\left(L_{1}\right)$ consists of the continuous spectrum $\sigma_{c}(L)=\left[U_{1},+\infty\right)$ and the point spectrum $\sigma_{p}\left(L_{1}\right)=\left\{\lambda_{1}, \ldots, \lambda_{M}\right\} \subset\left(0, U_{1}\right)$; all the eigenvalues $\lambda_{i}$ are of finite multiplicity and $M<+\infty$.

As usual, we set $\lambda_{M+1}=U_{1}$. Note that $H^{2}=H^{2}(\mathbb{R})$ and $H^{1}=H^{1}(\mathbb{R})$.

Proposition 7.4. Under assumption 7.2, the assumptions 2.6 and 2.7 are fulfilled.

Proof. We show only that assumption 2.6 holds, since the other part of the claim follows in the same way.

The continuous inclusion $H^{1}(\mathbb{R}) \subset L^{2+\sigma}(\mathbb{R})$ guarantees that $\Phi(u)$ is well defined and that

$$
F=\Phi^{\prime}: H^{1}(\mathbb{R}) \rightarrow H^{-1}(\mathbb{R}), \quad u \mapsto q(x)|u(x)|^{\sigma} u(x) .
$$

Hence, $\Phi \in C^{1}\left(H^{1}(\mathbb{R}) ; \mathbb{R}\right), \Phi(u) \geqslant 0$ and $\Phi(u)=0$ only if $u=0$. Moreover,

$$
\langle F(u), u\rangle=\int_{\mathbb{R}} q(x)|u(x)|^{2+\sigma} \mathrm{d} x=(2+\sigma) \Phi(u)
$$


and

$$
\|F(u)\|_{-1} \leqslant \text { const. }\langle F(u), u\rangle^{(1+\sigma) /(2+\sigma)} .
$$

Indeed, for $v \in C_{0}^{\infty}(\mathbb{R})$,

$$
\begin{aligned}
|\langle F(u), v\rangle| & =\left.\left|\int_{\mathbb{R}} q(x)\right| u(x)\right|^{\sigma} u(x) v(x) \mathrm{d} x\left|\leqslant \int_{\mathbb{R}}\right| q|u|^{\sigma} u|| v \mid \mathrm{d} x \\
& \leqslant\left[\int_{\mathbb{R}} q^{(2+\sigma) /(1+\sigma)}|u|^{2+\sigma} \mathrm{d} x\right]^{(1+\sigma) /(2+\sigma)}\left[\int_{\mathbb{R}}|v|^{2+\sigma} \mathrm{d} x\right]^{1 /(2+\sigma)} \\
& \leqslant|q|_{L^{\infty}}^{1 /(2+\sigma)}\langle F(u), u\rangle^{(1+\sigma) /(2+\sigma)} \text { const. }\|v\|_{1} .
\end{aligned}
$$

In order to fulfil assumption 2.8, we have to strengthen assumption 7.2 in the following way.

Assumption 7.5. Assumption 7.2 holds. Moreover,

(i) $q \in L^{(2+\sigma) /(\tau-\sigma)}(\mathbb{R})$,

(ii) there exists a constant $A>0$ such that $r(x) \geqslant A$ almost everywhere on $\mathbb{R}$.

We have now the following proposition.

Proposition 7.6. If assumption 7.5 holds, the assumption 2.9 is fulfilled.

Proof. These results are standard and can be found in [28] (except for the statement about the behaviour of $\Phi\left(u_{n}\right) /\left(\mu \Psi\left(u_{n}\right)\right)$ when $\left.\Psi\left(u_{n}\right) \rightarrow+\infty\right)$. Let us assume therefore that $\Psi\left(u_{n}\right) \rightarrow+\infty$, so that, in view of our assumptions, $\left|u_{n}\right|_{L^{2+\tau}} \rightarrow+\infty$. Then

$$
\begin{aligned}
& \Phi\left(u_{n}\right) \leqslant\left.\left.\frac{1}{2+\sigma}|q|_{L^{(2+\tau) /(\tau-\sigma)}}|| u_{n}\right|^{2+\sigma}\right|_{L^{(2+\tau) /(2+\sigma)}}=\frac{1}{2+\sigma}|q|_{L^{(2+\tau) /(\tau-\sigma)}\left|u_{n}\right|_{L^{2+\tau}}^{2+\sigma},} \\
& \Psi\left(u_{n}\right) \geqslant \frac{1}{2+\tau} A\left|u_{n}\right|_{L^{2+\tau}}^{2+\tau}
\end{aligned}
$$

give

$$
\frac{\Phi\left(u_{n}\right)}{\mu \Psi\left(u_{n}\right)} \leqslant \frac{2+\tau}{\mu(2+\sigma)} \frac{|q|_{L^{(2+\tau) /(\tau-\sigma)}}}{A}\left|u_{n}\right|_{L^{2+\tau}}^{\sigma-\tau} \rightarrow 0
$$

so we are done.

REMARK 7.7. It can be shown that we are in the situation described in remark 5.4. Indeed, if $\lambda \in] \lambda_{1}, U_{1}[$ and

$$
B_{\lambda}\left(u_{n}\right)=\int_{\mathbb{R}} u^{\prime}(x)^{2}+(V(x)-\lambda) u(x)^{2} \mathrm{~d} x \rightarrow-\infty,
$$

we have $B_{\lambda}\left(u_{n}\right) \geqslant-\lambda \int_{0}^{a} u_{n}(x)^{2} \mathrm{~d} x$, and since $\lambda_{1}>0$, we find, by the continuous inclusion $L^{2+\tau}(0, a) \subset L^{2}(0, a)$, that

$$
\int_{0}^{a}\left|u_{n}(x)\right|^{2+\tau} \mathrm{d} x \rightarrow \infty, \quad \Psi\left(u_{n}\right) \rightarrow \infty \quad \text { and } \quad B_{\lambda}\left(u_{n}\right) \geqslant-k \Psi\left(u_{n}\right)^{2 /(2+\tau)},
$$

where $k$ is a positive constant. 


\subsection{The non-existence of solutions}

Before stating the result of non-existence of solutions, we define what is meant by 'solution'. We say that $(\lambda, u) \in \mathbb{R} \times H^{1}(\mathbb{R})$ is a (weak) solution of the problem 1.1 if and only if

$$
J_{\lambda}^{\prime}(u)=0 \text { and } u \neq 0 .
$$

TheOREM 7.8. Suppose that the assumption 7.5 is fulfilled. Put

$$
c(\mu):=\left(\frac{\sigma}{\tau}\right)^{\sigma /(\tau-\sigma)} \frac{\tau-\sigma}{\tau}\left|\frac{q(x)^{\tau}}{r(x)^{\sigma}}\right|_{L^{\infty}}^{1 /(\tau-\sigma)} \mu^{-\sigma /(\tau-\sigma)} .
$$

Then problem 1.1 has no (weak) solutions $(\lambda, u) \in \mathbb{R} \times H^{1}(\mathbb{R})$ with $\lambda<\lambda_{1}-c(\mu)$.

Proof. It is sufficient to show that assumption 3.4 is fulfilled with the function $c(\mu)$ given in the proposition. So let us consider the function

$$
f(u):=\bar{q}|u|^{\sigma}-\mu \bar{r}|u|^{\tau}
$$

with $0<\sigma<\tau, \bar{q}>0, \bar{r}>0$ and $u \in \mathbb{R}$. A simple computation gives

$$
\max _{u \in \mathbb{R}} f(u)=\left(\frac{\sigma}{\tau}\right)^{\sigma /(\tau-\sigma)} \frac{\tau-\sigma}{\tau}\left(\frac{\bar{q}^{\tau}}{\bar{r}^{\sigma}}\right)^{1 /(\tau-\sigma)} \mu^{\sigma /(\tau-\sigma)} .
$$

Hence, we obtain

$$
\begin{array}{r}
(2+\sigma) \Phi(u)-(2+\tau) \Psi(u)=\int_{\mathbb{R}}\left(q|u|^{\sigma}-\mu r|u|^{\tau}\right) u^{2} \mathrm{~d} x \\
\leqslant\left(\frac{\sigma}{\tau}\right)^{\sigma /(\tau-\sigma)} \frac{\tau-\sigma}{\tau}\left|\frac{q(x)^{\tau}}{r(x)^{\sigma}}\right|_{L^{\infty}}^{1 /(\tau-\sigma)} \mu^{\sigma /(\tau-\sigma)}\|u\|^{2} .
\end{array}
$$

\subsection{A first solution set}

The results of $\S 5$ now give the following theorem.

THEOREM 7.9. Suppose that the assumption 7.5 holds and consider the problem 1.1 for $\lambda<\lambda_{1}$. Then

(i) there exists a non-decreasing, positive function $\mu_{1}(\lambda)$ defined on $\left(-\infty, \lambda_{1}\right)$ such that the problem 1.1 has no (weak) solutions for $\mu>\mu_{1}(\lambda)$,

(ii) there exists a non-decreasing, positive function $\bar{\mu}_{1}(\lambda)$ defined on $\left(-\infty, \lambda_{1}\right)$ with $\bar{\mu}_{1}(\lambda) \leqslant \mu_{1}(\lambda)$ such that the problem 1.1 has at least two pairs of (weak) solution pairs

$$
\left(\lambda, \pm v_{1, \lambda}\right) \text { and }\left(\lambda, \pm w_{1, \lambda}\right),
$$

provided that $0<\mu<\bar{\mu}_{1}(\lambda)$.

For $\mu>0$ fixed and such that $\mu<\lim _{\lambda \rightarrow \lambda_{1}^{-}} \bar{\mu}(\lambda)$, we set

$$
\Lambda_{1}:=\inf \left\{\lambda \mid \lambda<\lambda_{1} \text { and } \mu<\bar{\mu}_{1}(\lambda)\right\} \text {. }
$$




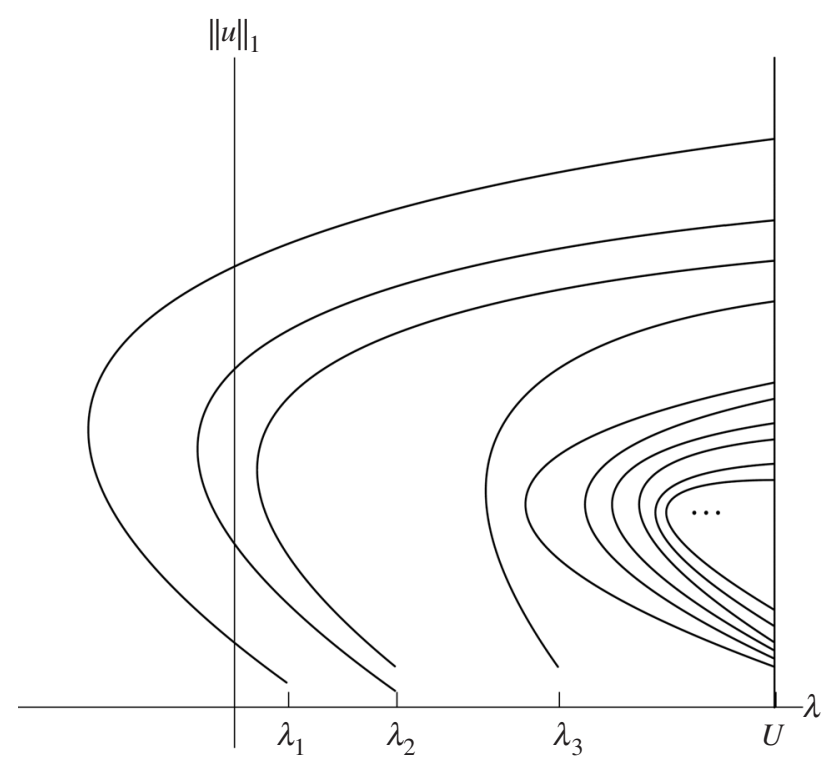

Figure 7. $\lambda_{1}$ and $\lambda_{3}$ are simple eigenvalues, $\lambda_{2}$ is a multiple eigenvalue and $M=3$.

Then the solutions $\left(\lambda, \pm v_{1, \lambda}\right)$ exist for $\lambda \in\left(\Lambda_{1}, \lambda_{1}\right)$ and the solutions $\left(\lambda, \pm w_{1, \lambda}\right)$ exist for $\lambda \in] \Lambda_{1}, U_{1}\left[\backslash \sigma_{p}\left(L_{1}\right)\right.$. We collect these solutions in a set $C_{1}$ which we call the first branch of solutions.

\subsection{The existence of other solution branches}

We remark that if assumption 7.5 holds, assumption 6.1 is fulfilled. Hence, we can apply the results of $\S 6$. We recall that $\sigma_{p}\left(L_{1}\right)=\left\{\lambda_{1}, \ldots, \lambda_{M}\right\}$ is the point spectrum of the linearization of problem 1.1 and that all the eigenvalues are of finite multiplicity. Remember that we have set $\lambda_{M+1}=U_{1}$ for the infimum of the continuous spectrum of this linearization and that $d(0)=0$ and $d(M+1)=+\infty$.

Theorem 7.10. Let $k_{0}$ be a fixed value belonging to the set $\{2, \ldots, M+1\}$. Then, for all $k \in\left\{d\left(k_{0}-1\right)+1, \ldots, d\left(k_{0}\right)\right\}$ (or $k>d(M)$ if $\left.k_{0}=M+1\right)$, there exists a non-decreasing, positive function $\bar{\mu}_{k}(\lambda)$ defined on $\left(-\infty, \lambda_{k_{0}}\right)$ with the following properties:

(1) $0<\bar{\mu}_{k+1}(\lambda) \leqslant \bar{\mu}_{k}(\lambda)$ on $\left(-\infty, \lambda_{k_{0}}\right)$;

(2) problem 1.1 has (at least) two solution pairs

$$
\left(\lambda, \pm v_{k, \lambda}\right) \text { and }\left(\lambda, \pm w_{k, \lambda}\right)
$$

provided that $0<\mu<\bar{\mu}_{k}(\lambda)$ and $\lambda \in\left(-\infty, \lambda_{k_{0}}\right) \backslash \sigma\left(L_{1}\right)$. These solutions are different for different values of $k$.

For $\mu>0$ fixed and such that $\mu<\lim _{\lambda \rightarrow \lambda_{k_{0}}} \bar{\mu}_{k}(\lambda)$ for some $k \in\left\{d\left(k_{0}-1\right)+\right.$ $\left.1, \ldots, d\left(k_{0}\right)\right\}$ (respectively, $k>d(M)$ ) we set

$$
\Lambda_{k}:=\inf \left\{\lambda \mid \lambda<\lambda_{k_{0}} \text { and } \mu<\bar{\mu}_{k}(\lambda)\right\} .
$$


The solutions $\left(\lambda, \pm v_{k, \lambda}\right)$ then exist for $\lambda \in\left(-\Lambda_{k}, \lambda_{k_{0}}\right) \backslash \sigma_{p}\left(L_{1}\right)$ and the solutions $\left(\lambda, \pm w_{k, \lambda}\right)$ exist for $\lambda \in\left(-\Lambda_{k}, U_{1}\right) \backslash \sigma_{p}\left(L_{1}\right)$. We collect these solutions in a set $C_{k}$ which we call the $k$ th branch of solutions.

When crossing with $\lambda$ an eigenvalue $\lambda_{k_{0}}$ from $\lambda_{k_{0}}-0$ to $\lambda_{k_{0}}+0$, it seems that $2 \operatorname{dim}\left(E\left(\lambda_{k_{0}}\right)\right)$ solutions cease to exist: at least we lose their variational characterization as pairs of solution pairs.

Figure 7 summarizes our results.

Let us make a final remark. The abstract problem developed above can be applied to more general situations including partial differential equations. But there are still open questions such as the one concerning bifurcation. All this will be the topic of our subsequent papers.

\section{References}

1 S. Alama and G. Tarantello. Elliptic problems with nonlinearities indefinite in sign. $J$. Funct. Analysis 141 (1996), 159-215.

2 A. Ambrosetti and P. H. Rabinowitz. Dual variational methods in critical point theory and applications. J. Funct. Analysis 14 (1973), 259-381.

3 T. Bartsch and Y. Ding. On a nonlinear Schrödinger equation with periodic potential. Math. Ann. 313 (1999), 15-37.

4 H. Berestycki and P. L. Lions. Nonlinear scalar field equations. II. Existence of infinitely many solutions. Arch. Ration. Mech. Analysis 82 (1983), 313-375.

5 D. M. Cao and X. P. Zhu. On the existence and nodal character of solutions of semilinear elliptic equations. Acta Math. Sci. 8 (1988), 345-359.

6 J. Chabrowski and J. Yang. On Schrödinger equation with periodic potential and critical Sobolev exponent. Topolog. Meth. Nonlin. Analysis 12 (1998), 245-261.

$7 \quad$ H.-P. Heinz. Lacunary bifurcation for operator equations and nonlinear boundary value problems on $\mathbb{R}^{N}$. Proc. R. Soc. Edinb. A 118 (1991), 237-270.

$8 \quad$ H.-P. Heinz. Existence and gap-bifurcation of multiple solutions to certain nonlinear eigenvalue problems. Nonlin. Analysis 21 (1993), 457-484.

9 H.-P. Heinz. On the number of solutions of nonlinear Schrödinger equations and on unique continuation. J. Diff. Eqns 116 (1995), 149-171.

10 H.-P. Heinz. Solutions of semilinear eigenvalue problems with corresponding eigenvalues in spectral gaps. J. Diff. Eqns Dynam. Syst. 4 (1996), 329-338.

11 H.-P. Heinz and C. A. Stuart. Solvability of nonlinear equations in spectral gaps of the linearization. Nonlin. Analysis 19 (1992), 145-165.

12 C. Jones and T. Küpper. On the infinitesimaly many solutions of a semilinear elliptic equation. SIAM J. Math. Analysis 17 (1986), 803-835.

13 W. Kryszewski and A. Szulkin. Generalized linking theorem with an application to a semilinear Schrödinger equation. Adv. Diff. Eqns 3 (1998), 441-472.

14 L. Landau and E. Lifschitz. Physique théorique ('Landau-Lifchitz'), vol. III: Mécanique quantique. Théorie non relativiste, 2nd edn (transl. E. Gloukhian) (Moscow: Éditions Mir, 1967).

15 A. A. Pankov and K. Pflüger. On a semilinear Schrödinger equation with periodic potential. Nonlin. Analysis 33 (1998), 593-609.

16 W. Rother. Nonlinear scalar field equations. Diff. Integ. Eqns 5 (1992), 777-792.

17 H.-J. Ruppen. The existence of infinitely many bifurcating branches. Proc. R. Soc. Edinb. A 101 (1985), 307-320.

18 H.-J. Ruppen. Le problème de Dirichlet non-linéaire sur $\mathbb{R}^{N}$. PhD thesis, Ecole Polytechnique Fédérale de Lausanne (1985). Thèse no. 593.

19 H.-J. Ruppen. Inherited bifurcation. Nonlin. Analysis 19 (1992), 993-1000.

20 H.-J. Ruppen. Nodal characterization of bifurcating branches in $L^{p}(\mathbb{R})$ for a semilinear equation. J. Diff. Eqns 99 (1992), 153-203.

21 H.-J. Ruppen. Multiplicity results for a semilinear, elliptic differential equation with conflicting nonlinearities. J. Diff. Eqns 147 (1998), 79-122. 
22 M. Struwe. Variational methods: applications to nonlinear partial differential equations and Hamiltonian systems (Springer, 1990).

23 C. A. Stuart. Bifurcation for variational problems when the linearisation has no eigenvalues. J. Funct. Analysis 38 (1980), 169-187.

24 C. A. Stuart. Bifurcation for Dirichlet problems without eigenvalues. Proc. Lond. Math. Soc. 45 (1982), 169-192.

25 C. A. Stuart. Bifurcation from the essential spectrum. In Proc. Western European Conf. on Differential Equations, Equadiff'82, Würzburg, 1982 (ed. H. Knobloch and K. Schmitt), Lecture Notes in Mathematics, vol. 1017 (Springer, 1983).

26 C. A. Stuart. A global branch of solutions to a semilinear equation on an unbounded interval. Proc. R. Soc. Edinb. A 101 (1985), 273-282.

27 C. A. Stuart. Bifurcation in $L^{p}(\mathbb{R})$ for a semilinear elliptic equation. J. Diff. Eqns 64 (1986), 294-316.

28 C. A. Stuart. Bifurcation in $L^{p}\left(\mathbb{R}^{N}\right)$ for a semilinear elliptic equation. Proc. Lond. Math. Soc. 57 (1988), 511-541.

29 C. Troestler and M. Willem. Nontrivial solution of a semilinear Schrödinger equation. Commun. PDEs 21 (1996), 1431-1449.

30 M. M. Vainberg (ed.). Variational methods for the study of nonlinear operators. (With a chapter on Newton's method by L. V. Kantorovich and G. P. Akilov. Transl. and suppl. A. Feinstein) (San Francisco, CA: Holden-Day Inc., 1964).

(Issued 14 October 2005) 